\title{
A process examination of the framing effect in younger and older adult medical decision making
}

\author{
Erin L. Woodhead \\ West Virginia University
}

Follow this and additional works at: https://researchrepository.wvu.edu/etd

\section{Recommended Citation}

Woodhead, Erin L., "A process examination of the framing effect in younger and older adult medical decision making" (2009). Graduate Theses, Dissertations, and Problem Reports. 2913.

https://researchrepository.wvu.edu/etd/2913

This Dissertation is protected by copyright and/or related rights. It has been brought to you by the The Research Repository @ WVU with permission from the rights-holder(s). You are free to use this Dissertation in any way that is permitted by the copyright and related rights legislation that applies to your use. For other uses you must obtain permission from the rights-holder(s) directly, unless additional rights are indicated by a Creative Commons license in the record and/ or on the work itself. This Dissertation has been accepted for inclusion in WVU Graduate Theses, Dissertations, and Problem Reports collection by an authorized administrator of The Research Repository @ WVU.

For more information, please contact researchrepository@mail.wvu.edu. 
A Process Examination of the Framing Effect in

Younger and Older Adult Medical Decision Making

Erin L. Woodhead, M.S.

\begin{abstract}
Dissertation
submitted to the Eberly College of Arts and Sciences at West Virginia University

in Partial Fulfillment of the Requirements for the degree of
\end{abstract}

Doctor of Philosophy

In

Psychology

Barry Edelstein, Ph.D., Chair

Rick Briggs, Ed.D.

Stanley Cohen, Ph.D.

Kevin Larkin, Ph.D.

B. Kent Parker, Ph.D.

Department of Psychology

Morgantown, WV

2008

Keywords: Heuristics, Framing Effect, Medical Decision Making, Think-Aloud 


\section{Abstract \\ A Process Examination of the Framing Effect in Younger and \\ Older Adult Medical Decision Making}

Erin L. Woodhead, M.S.

Older and younger adults are susceptible to a decisional bias when faced with medical decisions, which results in different treatment decisions when presented with survival or mortality data. Although this bias, termed the framing effect, has been demonstrated in multiple studies, no published studies have attempted to determine the decisional process older and younger adults engage in when presented with different information frames. The current study used a thinkaloud procedure to examine decisional process differences in younger and older adults who did and did not demonstrate a framing effect. All participants were presented with two data formats (interval and cumulative probabilities) in both survival and mortality wording. Data were analyzed quantitatively to determine the presence of a framing effect. Think-aloud data were analyzed qualitatively to determine decisional process differences stratified by age and demonstration of the framing effect. Interpretation of results for the interval probability format was limited by typographical errors that were found to exist in the scenario after data collection was complete. No statistically significant framing effects were found for either format among both younger and older adults. Older and younger adults had relatively equal personal and vicarious experience with the decision. Qualitative analyses performed on a subset of the data suggest that, among those older adults who did not demonstrate the framing effect, there was a reliance on pertinent experience with the decision, and little reliance on the presented data. In contrast, among younger adults who demonstrated the framing effect, there was a tendency toward incomplete analysis of the data when younger adults had knowledge about the decision. Across age groups, those that demonstrated the framing effect were significantly more likely to reference the presented data. Older adults were significantly less likely to reference the presented data than younger adults. Younger and older adults did not differ significantly on timeto-decision or word count for the think-aloud transcripts. Results are discussed in terms of agerelated differences in decision making processes. 


\section{Acknowledgements}

I was very lucky throughout this project to benefit from the guidance and help of many individuals. I am very appreciative of my advisor and chair, Barry Edelstein, Ph.D., who helped me figure out how to design a rigorous qualitative study, and who has generally been awesome throughout my entire graduate school career. Justin Timins, Christine Weir, Kayla Williams, Heidi Storrick, and Salena Garced were my dedicated research assistants throughout the project and were tremendously helpful with the qualitative analyses. Sarah Rudy, Kat McLaughlin, Donovan Sarr, and Zach Murray volunteered their time helping with interrater reliability and providing feedback on our qualitative categories, for which I am very grateful. I would also like to thank the staff and community members at the Greene County and Washington County senior centers in Pennsylvania, who were very gracious with their facilities and time when I was recruiting and running participants. The other members of my committee, including Rick Briggs, Ed.D., Stan Cohen, Ph.D., B. Kent Parker, Ph.D., Kevin Larkin, Ph.D., were a great help in refining the project, and I really appreciated their support of the qualitative aspect of the study. I also appreciate the support of my family and fiancé. 
Table of Contents

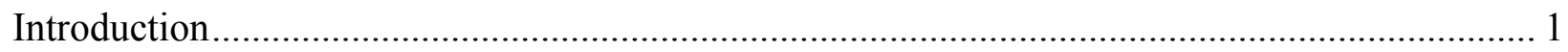

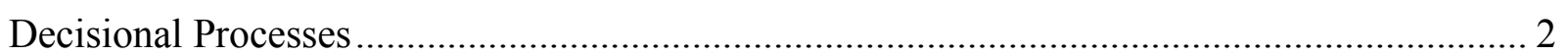

Everyday Problem Solving ........................................................................................ 3

Decisional Biases ..................................................................................................... 7

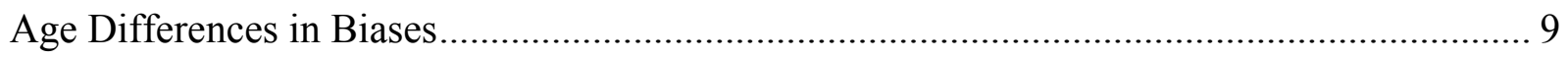

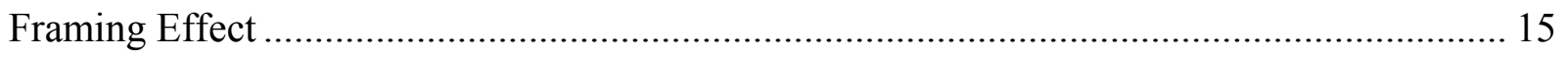

Framing Effect \& Medical Decision Making ................................................................ 17

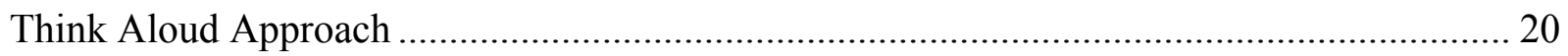

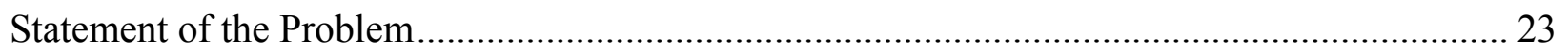

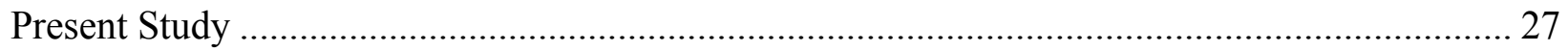

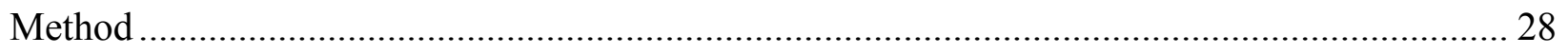

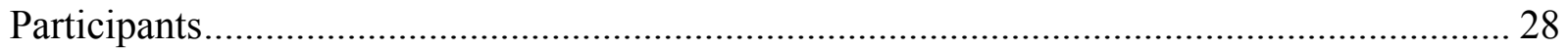

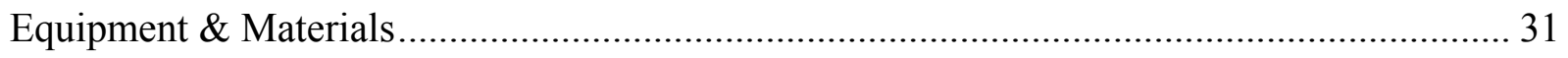

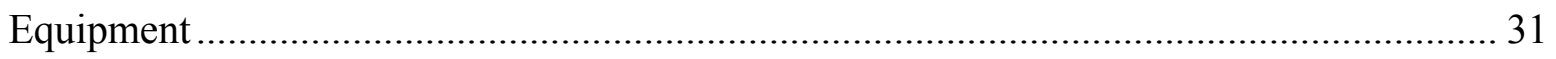

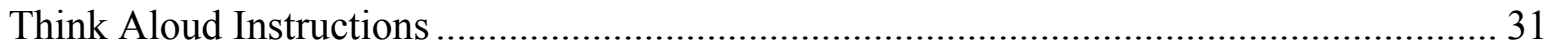

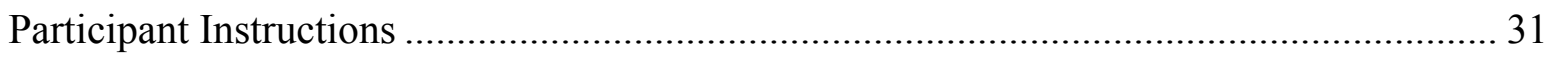

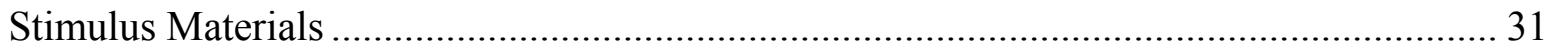

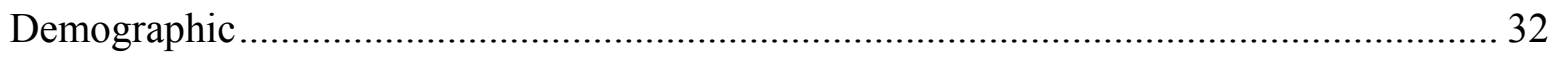

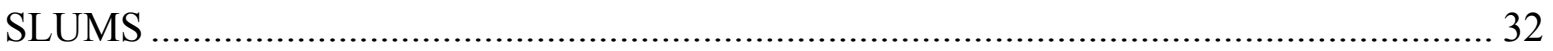

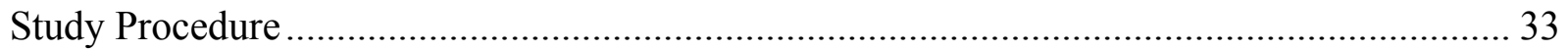

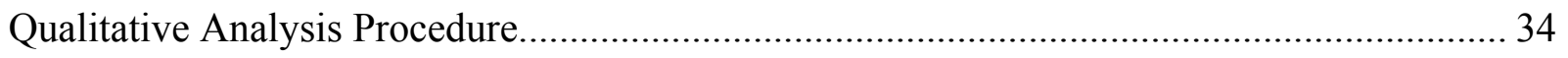

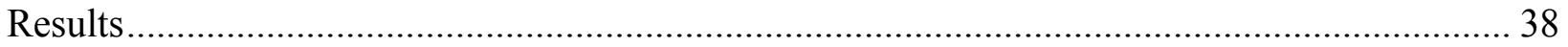




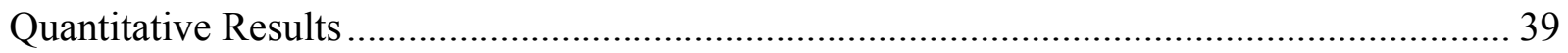

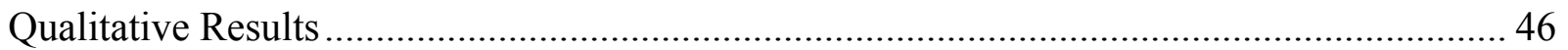

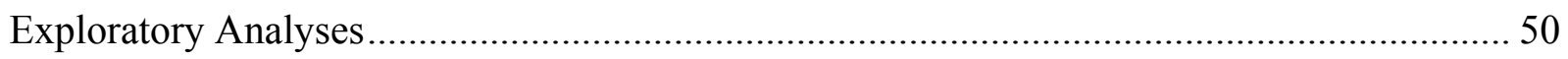

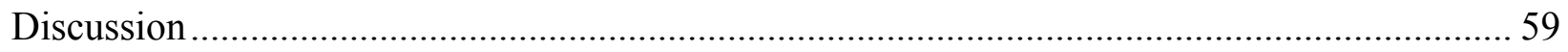

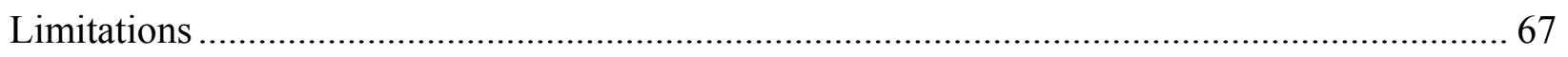

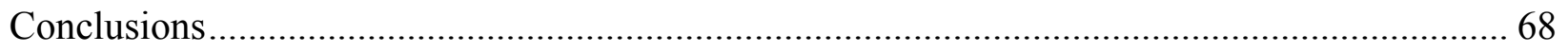

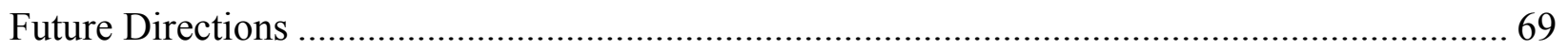

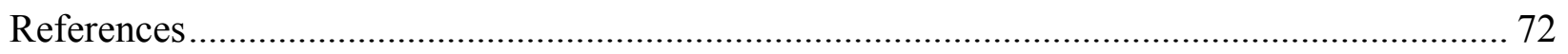

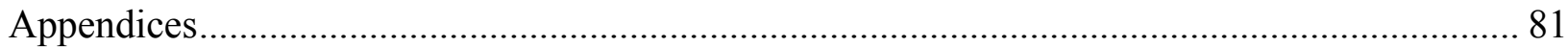




\section{List of Tables}

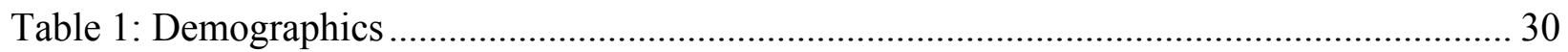

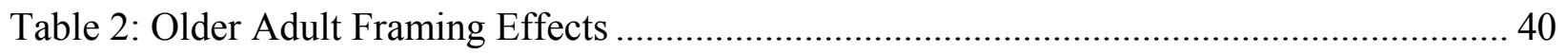

Table 3: Younger Adult Framing Effects ..................................................................... 42

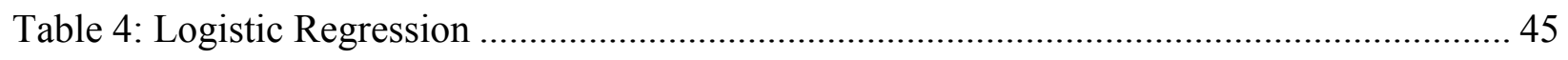

Table 5: Word Count \& Decision Time......................................................................... 51

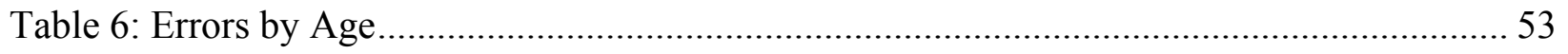

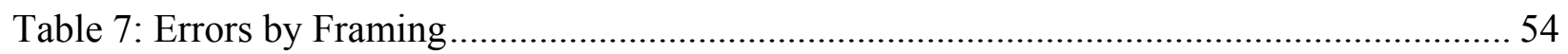




\section{A Process Examination of the Framing Effect in}

\section{Younger and Older Adult Medical Decision Making}

The population of older adults is steadily increasing. In 2002, those over the age of 65 comprised over 12 percent of the U.S. population. By the year 2030 the percentage of the U.S. population over the age of 65 is projected to jump 20 percent (U.S. Administration on Aging, 2003). Although approximately 38 percent of non-institutionalized older adults report their health as being "excellent" or "very good" (U.S. Administration on Aging, 2003), approximately 88 percent of older adults report having at least one chronic medical condition (King, Rejeski, \& Buchner, 1998).

As one ages, the increased risk of disease leads to the increased demand to make important medical decisions. Additionally, with increasing age, the likelihood that one will face long term care increases, where decisions are often made regarding medical advance directives. Four out of every ten people over the age of 65 will use a nursing home at some point in their lives, and about 20 percent of nursing home users will spend five or more years in the nursing home (Centers for Disease Control and Prevention, 2002). Decisions regarding advance directives are not made only by those considering long-term care. The Patient SelfDetermination Act (PSDA) requires that hospitals, nursing homes, home health organizations, and most HMOs provide patients with a statement regarding their health care decision making rights, and document whether the patient has an advanced directive (American Cancer Society, 2005). The PSDA encourages all patients to make medical decisions for future care. Given the high rate of chronic disease in older adults, it is probable that this is the population most affected by the PSDA. As an example of the impact of the PSDA, the Act has increased the rate of advanced directives among nursing home residents from 4.7 to 34.7 percent (Bradley, Wetle, \& 
Horwitz, 1998). Therefore, the number of medical decisions increase with age, whether for current medical problems or in preparation for future medical problems. Moreover, as one ages, the likelihood of facing potentially life threatening decisions increases. In spite of the potential impact of medical decisions, we know little about how older adults make their medical decisions. Additionally, we know little about how the decision processes used among older adults differ from those used among younger adults.

\section{Examination of Decisional Process}

There is limited literature that has addressed medical decision making processes and quality among older adults. There is some evidence that older adults take less time to make medical decisions than younger adults (Leventhal, Leventhal, Schaefer, \& Easterling, 1993), seek less information about treatment alternatives (Meyer, Russo, \& Talbot, 1995), and show less evidence of complex comparison and evaluation of the information available to them (Zwahr, Park, \& Shifren, 1999). Much of the research that has examined the decisional process among older adults has focused on problem solving abilities and deficits in this population. Because decision making and problem solving are essentially two parallel but related literatures, it is necessary to briefly consider the similarities and differences between decision making and problem solving. Some authors have argued that problem solving and decision making are more alike than different (e.g., Thornton \& Dumke, 2005), and that they share the same foundation of goal-directed cognition (Marsiske \& Margrett, 2006). However, problem solving focuses on the outcomes and effectiveness of generated solutions, whereas decision making examines how individuals evaluate multiple alternatives and choices (Marsiske \& Margrett, 2006).

D'Zurilla (1986) defined problem solving as “the process by which an individual or group discovers a solution to a problem" (p. 13). D’Zurilla conceptualized decision making 
somewhat differently: "the purpose is to evaluate the available solution alternatives and to select the 'best' one(s) for implementation in the problematic situation"' (p. 34). Problem solving often requires multiple steps, including problem orientation, problem definition and formulation, generation of alternative solutions, decision making, and solution implementation and verification (D’Zurilla \& Nezu, 1982). More recently Finucane, Slovic, Hubbard, Peters, Mertz, and MacGregor (2002) distinguished between problem solving and decision making in stating that problem solving involves "assessing the present and desired state of affairs and finding ways to move from the former to the latter" whereas decision making involves, "evaluating the possible solutions and selecting one to use" (p. 142).

Taken together, the differences between problem solving and decision making, respectively, include: focus on outcomes vs. focus on process, generation of alternatives vs. provision of potential solutions, need to define the problem vs. problem provided for the individual, and focus on instrumental tasks (e.g., self and home maintenance) vs. focus on relatively low frequency decisions (e.g., buying a car, making a major medical decision). Decision making might therefore be seen as one type of problem solving, albeit a type where solutions are already presented to the individual.

\section{Everyday Problem Solving}

Decisional processes among older adults have largely been examined in the context of everyday problem solving. The everyday problem solving and decision making literatures emphasize different processes and outcomes, and often ask different questions. Nevertheless, everyday problem solving researchers have contributed to our understanding of older adult decision making through the examination of the decision making process and the strategies used 
to solve problems. Therefore, we can draw on the everyday problem solving literature to better understand how older adults might be approaching medical decisions.

\section{Age-Related Differences in Everyday Problem Solving}

Although a recent meta-analysis found that younger adults outperformed older adults on everyday problem solving tasks (Thornton \& Dumke, 2005), other researchers have found that age differences are present in some elements of problem solving but not others. For example, some researchers have found that older adults seek less information than younger adults when solving problems (e.g., Berg, Johnson, Meegan, \& Strough, 2003; Meyer et al., 1995), and make their decisions more quickly than younger adults (Meyer et al., 1995). Several researchers have found that, although older adults may be seeking less information and taking less time to make their decision, their solutions are similar to solutions produced by younger adults. Thus, such decisional strategies as taking less time and seeking less information may not negatively impact the final outcome (e.g., Walker, Fain, Fisk, \& McGuire, 1997). Therefore, there is some research indicating that older adults may be making decisions that are less informed than younger adults, by not considering all the pieces of information in a decision task, and some research indicating that the final solutions produced by older adults are as effective as solutions produced by younger adults.

Researchers who have focused on the relatively poorer performance of older adults on decision making and problem solving tasks have accounted for age differences on the basis of age related changes in basic cognitive skills. In general, normal aging involves a decline in abilities related to fluid intelligence, such as spatial orientation, perceptual speed, and numeric ability. However, these declines are modest in most healthy older adults until about age 80 , when more significant declines may be seen. Other cognitive skills that are strongly related to 
education, such as verbal ability, have been found to remain intact in healthy adults until well into older adulthood (c.f., Schaie, 1994). These normative changes potentially have differential effects on older adults' ability to engage in everyday problem solving tasks. Allaire and Marsiske (1999) found strong relations between measures of everyday problem solving ability and traditional measures of reasoning and working memory, indicating that everyday problem solving ability may decline because it relies on the same basic abilities that decline with age (e.g., fluid intelligence). Marsiske and Willis (1995) proposed that everyday problem solving is a complex "compiled" form of cognition. Therefore, the decline seen in everyday problem solving among older adults is consistent with the decline seen among older adults in basic cognitive abilities such as fluid intelligence (Schaie \& Willis, 1996).

Other researchers have accounted for the equality in problem solving performance between older and younger adults by appealing to the notion of accumulated problem solving expertise over the lifespan (Finucane et al., 2002). For example, Walker et al., (1997) found that in a driving decision task, the overall quality of younger and older adults' route decisions was similar. Additionally, Meyer et al. (1995) found that, although older adult women sought less information about the decision, the outcome of their decisions (treatment for breast cancer) were equivalent to the outcomes of the decisions made by the younger adult women. Therefore, older adults may compensate for age-related cognitive decline by relying on their accumulated problem solving expertise. Relying on this expertise produces decisions that are equivalent to younger adult decisions, and produces solutions to problems that are as effective as solutions produced by younger adults, who are presumably relying more heavily on cognitive resources during the problem solving task. 


\section{Problem Content}

As noted earlier, much of the research on age differences in decisional quality has been examined in the everyday problem solving literature. This literature has examined a wide variety of everyday problems, including interpersonal problems, such as resolving conflicts with friends (e.g., Blanchard-Fields, Mienaltowski, \& Seay, 2007; Cornelius \& Caspi, 1987), and instrumental problems, such as food preparation, medication use, and telephone use (e.g., Diehl, Willis, \& Schaie, 1995). Additional problems that have been examined include interpreting maps, charts, and tables (e.g., Willis \& Schaie, 1986), and planning driving routes (e.g., Walker et al., 1997). Researchers in this area have found that older adults choose more effective strategies than younger adults for solving interpersonal tasks, as rated by a panel of judges (e.g., Blanchard-Fields et al., 2007; Thornton \& Dumke, 2005). Several researchers (e.g., Willis \& Schaie, 1986) have found that performance on everyday instrumental tasks was predicted by various primary abilities, such as figural relations, inductive reasoning, and verbal analogies. Therefore, it appears that on instrumental tasks, performance is significantly tied to fluid intelligence, which declines with age. Taken together, the everyday problem solving literature has examined problems that occur regularly for older adults, and that capitalize on problem solving expertise that older adults have accumulated throughout their lifespan. One conclusion that can be drawn from an examination of problem content is that older adults appear to be producing more effective strategies for interpersonal problems than instrumental problems.

One area that has been neglected in the problem solving literature is medical treatment decisions. Medical decisions are potentially more salient for older adults, and are potentially faced as frequently as tasks identified as "everyday problems." The everyday problem solving literature has also largely ignored the influence of decisional biases on the decision maker. 
Decision making researchers have devoted a large amount of research to the topic of decisional biases, including who is most susceptible to biases and the conditions under which they are most likely to occur. Additionally, a substantial amount of research has examined the influence of decisional biases on medical decisions. Therefore, although the everyday problem solving literature has provided a framework for how to examine older adults' decisional quality and process, it has not examined how older adults approach medical decisions or how their approaches may lead to decisional biases. An examination of biases is particularly important, as it is possible that older adults' worse performance on decisional tasks is due to biases in the decision making process. There is a substantial amount of research indicating that adult decision makers of all ages are susceptible to decisional biases in the process of making a decision, and that these biases impact medical decisions.

\section{Decisional Biases}

In a classic article on decision making, Tversky and Kahneman (1974) described three of the most common heuristics, or mental shortcuts, individuals use when making a decision: the representativeness heuristic, the availability heuristic, and the adjustment and anchoring heuristic. Each of these heuristics can lead to biased or poorly informed decisions. Tversky and Kahneman (1974) demonstrated the representativeness heuristic by giving participants brief personality descriptions of several individuals. The participants were then asked to assess the probability that each of these individuals held a certain occupation, based on a list of occupations presented to participants. A sample description was: "Steve is very shy and withdrawn, invariably helpful, but with little interest in people, or in the world of reality. A meek and tidy soul, he has a need for order and structure, and a passion for detail” (Tversky \& Kahneman, 1974, p.1124). Participants were most likely to choose librarian as the most probable occupation 
for Steve. This judgment was presumably based on how representative Steve was of the stereotype the participants held about librarians (e.g., reserved and quiet), instead of basing the decision on facts that could inform the decision (e.g., the number of libraries in the area).

The availability heuristic is demonstrated with an example from Hastie and Dawes (2001). When asked which is more common, murder or suicide, individuals often reply that murder is more common, even though statistics indicate that suicide is more common. The answer of murder is presumably given because murders often get more publicity than suicides, and therefore this information is more "available" than information on suicides.

In an example of the third heuristic, adjustment and anchoring, Tversky and Kahneman (1974) asked participants to provide answers to various questions, and to state their answers in percentages. For example, participants were asked to estimate the percentage of African countries in the United Nations. Prior to giving their answer, participants were given a random number, generated by spinning a wheel, between 1 and 100 (the anchor). The participants were then asked to estimate the answer to the initial question. For those participants initially given the number 10 as their anchor, the median answer to the original question was 25 percent. For those participants initially given the number 65 as their anchor, the median answer to the original question was 45 percent. This example illustrates that the presentation of a high or low anchor is related to the answer that is provided.

There are a number of ways in which these decisional biases might impact medical decisions. For instance, the availability heuristic involves decision making based upon the information that is most "available" to us in our long-term memory (Tversky \& Kahneman, 1973), as opposed to considering all the pieces of information that might inform one's final decision. Thus, the decision is biased by the selective consideration of available information, 
which may adversely affect choice for a medical treatment. Likewise, use of the availability heuristic can lead us to ignore base-rate information, or how often a medical problem occurs in the general population. Participants asked to estimate the likelihood of a rare disease often ignore base-rate information, or use the information inappropriately, therefore producing a bias in their final decision (Medin \& Edelson, 1988).

\section{Age Differences in Decisional Biases}

Although adult decisions makers of all ages are susceptible to decisional biases, there are several researchers who have argued that older adults are more likely to be susceptible to decisional biases than younger adults (e.g., Park, 1999; Yates \& Patalano, 1999). Yates and Patalano (1999) support their argument by explaining three modes of decision making: analytic, rule-based, and automatic. Individuals move through the decision making modes in a linear fashion. Upon first encountering a decision, one must invoke the analytic mode, as the presented decision is unfamiliar and there is little previous data on which to base the decision. Upon encountering the problem multiple times, rules are developed for how to handle the situation. In this stage, referred to as rule-based decision making, a deliberative judgment is made regarding the next action. After the rules have been applied consistently, the decision becomes automatic, and thus a heuristic is formed, which is relied on without deliberate judgment. Therefore, it is possible that both the rule-based and automatic decision modes could result in biased decision making.

The analytic mode places heavy demands on working memory. Thus, Yates and Patalano (1999) argued that age differences in mode use will emerge due to normal age-related cognitive decline in working memory. Numerous studies have found decreases in working memory with increasing age (e.g., Salthouse \& Babcock, 1991). Decreases in working memory coupled with 
the slowing of cognitive operations in older adults negatively impacts performance on complex tasks (e.g., Salthouse, 1992, 1996). The type of complex tasks on which older adults perform worse due to age-related cognitive decline are similar to the tasks required in analytic decision making. Thus, due to normative cognitive declines, Yates and Patalano (1999) argued that older adults will be more likely to use the automatic decision mode, which potentially involves a greater reliance on heuristics.

It is possible that older adults might compensate for their cognitive declines by relying on rules that are based on prior knowledge and/or personal experience with the decision. This is also problematic if one adopts Yates' decision modes, as it is likely that a reliance on rules based on personal information leads to heavy reliance on heuristics. Pierce (1993) supported this assertion when she found that patients who had personal experience with the presented decision (breast cancer treatment) made treatment decisions rapidly, before hearing all possible alternatives. This approach to decision making is sometimes termed "satisficing," (Simon, 1957) meaning that the participants evaluated alternatives until they found the first option that met their standards, at which point they chose that option and stopped evaluating additional options.

Some researchers have found that the accumulated knowledge and experience of older adults' might reduce biased decision making. For instance, Zwahr (1994) found that what female participants knew about menopause and estrogen replacement therapy was positively related to how many treatment options they perceived as being available to them, how frequently comparisons were made among treatment alternatives, and how well participants supported their chosen alternative. Thus, participants had personal knowledge about the treatment, which potentially led them to consider the presented information in light of their knowledge. 
Therefore, this study provides evidence that, at least under some conditions, accumulated knowledge does not necessarily lead to an automatic decision making mode.

There is also research to support the argument that older adults' acquired knowledge about decision making protects them against more traditional decisional biases, such as the attraction effect. The attraction effect occurs when the addition of an irrelevant option into a set of options increases the proportion of individuals who choose the irrelevant option. Kim and Hasher (2005) found that older adults were less susceptible to the attraction effect than younger adults in both an extra credit task and grocery shopping task, even though the older adult participants rated themselves as having less knowledge than the younger adults about the extra credit task. The authors' explain their results as evidence that the accumulated number of decisions that older adults have made in their lifetime compensates for their presumed cognitive declines, and therefore the presumed cognitive decline may not necessarily lead to biased decision making, as other authors have proposed (e.g., Yates \& Patalano, 1999).

Although the assertion that accumulated knowledge protects older adults from decisional biases is supported by recent research, there is a larger amount of literature indicating that older adults are more susceptible to decisional biases than younger adults across multiple domains. Riggle and Johnson (1996) used process tracing techniques to determine whether older and younger adult participants were engaging in satisficing. Younger and older adult participants were presented with a matrix including candidate names and key issues. They could freely access any cell, which would elaborate on how a particular candidate felt about the key issue selected. The participants were then asked to choose a political candidate based on the information they had received. Riggle and Johnson found that older adults took longer to complete their information search and decision than younger adults, and that the older adults 
spent longer examining each piece of information. The older adult participants also accessed significantly fewer cells than the younger adult participants. To examine susceptibility to heuristics, the researchers analyzed each participant's search strategy, based on what pieces of information they had accessed. They found that the older adult participants were more likely to engage in "satisficing," meaning that they searched the information by candidate until they found the first acceptable candidate who met their standards. Therefore, the older adults' decisions were potentially biased due to their reliance on satisficing.

Another example of older adults' susceptibility to traditional decisional biases can be found in a study by Spaniol and Bayen (2005), which examined age differences in judgments of conditional probability. The judgment task required participants to judge the probability that an individual had a disease in light of specific symptoms. Participants were presented with descriptions of 80 patients who each had one of eight symptoms that were characteristic of one of two fictitious diseases. After these associations were presented for a period of time, participants were presented with a new group of patients who had one of the same eight symptoms presented in the first phase. Participants were asked to diagnose each patient with one of the two fictitious disease, and estimate the probability that the diagnosis was correct. Participants were allowed to assign their "probability score" anywhere between 50 percent and 100 percent. A probability score of one-hundred percent meant that they were completely confident in their diagnosis, whereas a 50 percent score indicated that they were not at all certain of the diagnosis. Base rates were not provided for each fictitious disease. Rather, participants were expected to learn the typical combinations of symptoms and disease pairings from the first set of associations. A second component of the study (frequency judgment block) presented participants with 40 patients with one of the eight symptoms. These symptoms were not 
associated with a diagnosis, and were all different from the symptoms presented in the first component. The eight symptoms were assigned a frequency score of 1 to 9 . Following presentation of the 40 patients, participants were asked to estimate how many patients had each of the symptoms. They were told that the correct number was between 1 and 9 .

Diagnoses for each age group were examined for proportion correct, and over and under confidence. Proportion correct was defined as the proportion of disease diagnoses in which the participant chose the diagnosis that was most likely given the symptoms. Over confidence was defined as a tendency for the probability judgments made by the participants to reflect an overestimation of the accuracy of their diagnosis. Conversely, under confidence was defined as a tendency for the probability judgments made by the participants to reflect an underestimation of the accuracy of their diagnosis.

The results indicated that, when older and younger adults were given the same amount of time to examine the stimulus materials, frequency judgments of how many patients showed each of the eight symptoms were less accurate among older than younger adults. Proportion of correct diagnoses among older adults was significantly lower than among younger adults, and older adult participants scored significantly lower on the researchers' measure of under confidence. Therefore, older adults were more susceptible to errors in judgments of conditional probability when presented with hypothetical medical decisions about fictitious patients and diseases.

As another example of susceptibility to decisional biases in older adults, Chen and Sun (2003) evaluated the use of a "single deal strategy" and a "multiple deal strategy" among younger and older adults asked to engage in a yard-sale decision making task. Younger and older participants were presented sequentially with 120 items to be sold in a yard sale. They were given three offers for each item, and had to choose the best offer. The offers were 
presented one at a time, and once an offer was rejected, it could not be reconsidered. The participants' working memory capacity was assessed using a reading span task. The authors used a computer simulation to determine that a "single deal strategy" required significantly less reliance on working memory. In this strategy, the first offer is always rejected. If the second offer is better than the first, it is selected. If it is not better than the first, it is rejected and the third offer is selected. This strategy requires the participant to keep only one other offer in shortterm memory. This was compared to the "multiple deal strategy" which required the participant to remember the offers from previous trials and use those offers to compare against the current offer. Therefore, in this strategy, it might be beneficial to take the first offer on a new yard sale item, regardless of what item is for sale, if the first offer is significantly better than all previous offers on similar items. This strategy thus requires the participant to remember all previous offers. Chen and Sun (2003) found that older adults were significantly more likely than the younger adults to use the single deal strategy, and the younger adults were more likely to use the multiple deal strategy. The authors concluded that the older adults' reduced working memory capacity forced them to engage in the heuristic of the single deal strategy. Interestingly, older and younger adults earned the same amount of money overall from the yard-sale tasks. Therefore, the use of a single deal strategy apparently did not negatively impact the final outcome.

The above research indicates that older adults are susceptible to traditional decisional biases. Although there is some research to support the notion that older adults are protected from decisional biases due to accumulated experience and expertise in decision making (Kim \& Hasher, 2005), the majority of the research indicates that older adults have an increased level of biased reasoning as compared to younger adults, although these biases do not necessarily 
contribute to different outcomes (e.g., Chen \& Sun, 2003). Research from the everyday problem solving literature indicates that older adults are sometimes performing as well as younger adults, though they are using different strategies (e.g., Walker et al., 1997), and that they are sometimes performing worse than younger adults on instrumental problem solving tasks (Thornton \& Dumke, 2005). Therefore, both the problem solving and decision making literatures indicate that older adults are engaging in different strategies than younger adults to reach their final decision, but that these strategies are not consistently detrimental to the final outcome. However, one such decisional bias that has been shown to be detrimental to the final outcome of decision making tasks among older and younger adults is evidenced by the framing effect.

\section{The Framing Effect}

The framing effect has been examined with younger (Almashat, Ayotte, Edelstein, \& Margrett, 2008; Tengs, 1987) and older adults (e.g., Kim, Goldstein, Hasher, \& Zacks, 2005; McKee, 2001; Schuller, 2006), and has particularly significant implications for older adults' health and welfare. The framing effect occurs when an individual is asked to make a choice between two options that are presented in either positively or negatively "framed" scenarios, and subsequently makes different choices when deciding between the options. One option is often presented as a "risk-seeking" option and one is presented as a "risk-averse" option. Formally defined, the framing effect is a "significant difference observed in subjects' responses to $F_{+}$ (positive frame) and F. (negative frame; Piñon \& Gambara, 2005, p. 325).”

The framing effect has been studied across a variety of domains (see Kühberger, 1998 for review). In the classic framing effect problem, termed the "Asian Disease" design, participants were asked to choose between two options, with one option representing a risk-seeking option, and the other representing a risk-averse option. This design is primarily based on Tversky and 
Kahneman's (1981) landmark study on the framing effect. Participants were asked to choose one of two treatment options for an outbreak of a fatal "Asian disease." The information was framed in either a "lives saved" (positive) manner or a "lives lost" (negative) manner. The scenarios are presented below:

Lives Saved Frame

If Program A is adopted, 200 people will be saved (risk-averse option). If Program B is adopted, there is a one-third probability that 600 people will be saved and a two-thirds probability that no people will be saved (riskseeking option).

\section{Lives Lost Frame}

If Program C is adopted, 400 people will die (risk-averse option). If Program D is adopted, there is a one-third probability that nobody will die and a two-thirds probability that 600 people will die (riskseeking option; Tversky \& Kahneman, 1981, p. 453).

Participants were more likely to choose the risk-averse option (Program A) when presented with the "lives saved" frame, and choose the risk-seeking option (Program D) when presented with the "lives lost" frame. Therefore, it appears that in the "lives saved" frame, participants were not willing to take a chance on a risky program and preferred to "play it safe," whereas the "lives lost" frame induced a more risky attitude, and a willingness to take a gamble on the possibility of saving more lives. Thus, the change of frame produced significantly different decisions. The inconsistency produced by the framing effect is particularly troubling when one examines how pervasive the framing effect is across domains, particularly in domains where decisions may be life-threatening, such as in the area of medical decision making. 
The Framing Effect and Medical Decision Making

The first published study that examined the influence of frame on medical decision making (McNeil, Pauker, Sox, \& Tversky, 1982) found that this effect was present when participants were asked to choose between hypothetical treatments for lung cancer. Participants were presented with hypothetical scenarios, framed in either survival or mortality rates, and asked to choose between radiation or surgery treatment for lung cancer. The format of the data was also manipulated, such that participants received both a cumulative probability format (e.g., 10 will die in treatment, 32 will have died by 1 year) and an overall life expectancy format (e.g., the number of people who live 1 to 5 years after treatment). Participants were more likely to choose surgery, the high-risk option with higher long-term survival rates, when information was presented in terms of survival, and choose radiation, the low-risk option with higher short-term survival rates, when information was presented in terms of mortality. This effect was present in both the overall life expectancy data format and the cumulative probability data format.

Therefore, in these data formats, participants deciding between gains (survival frame) were riskseeking, whereas those deciding between losses (mortality frame) were risk-averse.

Tengs (1987) added an interval probability format to the scenarios used by McNeil et al. (1982). Undergraduate participants received either survival or mortality frames in three data formats (cumulative probability, interval probability, and overall life expectancy). A framing effect was found with the cumulative probability format, where participants chose surgery more often in the survival frame and radiation more often in the mortality frame. Tengs found a similar pattern of responses as McNeil et al. (1982): participants more often chose surgery (the high-risk option) in the survival frame and radiation (the low-risk option) in the mortality frame. Therefore, both the outcome information type (cumulative probability, interval probability, or 
life-expectancy) and the frame (survival or mortality) had an impact on the medical treatment chosen.

McKee (2001) studied the framing effect among older and younger adults using the same lung cancer scenarios as McNeil et al. (1982), which asked participants to choose between radiation or surgery for treatment of lung cancer. Scenarios were framed either in terms of survival or mortality rates. Both younger and older adults were susceptible to the framing effect when information was presented in an overall life expectancy format, as indicated by choosing surgery more often in the survival frame and radiation more often in the mortality frame. However, older adult participants demonstrated the framing effect in fewer comparisons than did the younger adult participants.

Kim et al. (2005) examined the framing effect among younger and older adults using the cumulative probability format from the McNeil et al. (1982) study. Participants were asked to either read the scenario and make their decision (surgery or radiation), or they were asked to read the scenario, make their decision, and then justify it. Younger adults did not exhibit the framing effect in either condition. For older adults the framing effect was demonstrated when not asked to justify their decision with the cancer scenario. The framing effect was precluded with the group that justified their decisions. Thus, in addition to demonstrating the framing effect among older adults, this study also attempted to preclude the framing effect.

In a demonstration of the framing effect among younger adults, Almashat et al. (2008) used the same six medical decision scenarios used by Tengs (1987) and McKee (2001). Undergraduate participants were either presented with survival or mortality worded scenarios, with one scenario presented in cumulative probability format, one in interval probability format, and one in overall life-expectancy format. Before making their choices, half of the participants 
received a debiasing questionnaire asking them to consider the advantages and disadvantages of surgery vs. radiation. The other participants (control group) received a questionnaire containing general information related to cancer. When the data were presented in cumulative probability format, participants more often chose surgery in the survival worded scenario and radiation in the mortality worded scenario. Therefore, a framing effect was found for the cumulative probability format, as indicated by the discrepancy in treatment choice based on survival or mortality wording. A framing effect was not found for the scenarios that were worded in interval probability format or overall life expectancy format.

In another demonstration of the framing effect, Schuller (2006) examined age differences in a breast cancer scenario, adapted from the "Asian Disease" design. Participants were asked to rate their decision on a Likert type scale. Both frames (lives saved and lives lost) were presented to all participants. The treatment decisions of young women were not affected by frame, but older women who received the negative scenario first were more likely to make risky decisions across all scenarios.

Finally, Woodhead (2006) examined the framing effect among younger and older adults. Participants received a cumulative probability, interval probability, and overall life expectancy format, in either survival or mortality wording. Younger adults exhibited the framing effect in the cumulative and interval probability formats. Older adults exhibited the framing effect with the interval probability format.

The results of these studies indicate that patients of all ages may not be making bias-free decisions when presented with a few options for treatment, given that respondents make different decisions when the information is presented in various frames and outcome information types. Patients may potentially choose a more risky option when the information is presented in terms 
of survival rates. Additionally, how the outcome information is presented (cumulative probability, interval probability, or life-expectancy) has an effect on the final decision made by a patient.

In light of the many studies showing support for a framing effect in medical decision making, it is critical to know exactly what pieces of information participants are considering when making a hypothetical medical decision. Additionally, it is crucial to understand how one considers the presented information and whether that directly relates to demonstration of decisional biases, namely the framing effect. One way to understand what individuals are considering when making a medical decision is to ask them to verbalize their decisional process.

\section{Think-Aloud Approach to Decision Making}

One method for eliciting participants' strategy in a decision making or judgment task is to ask them to "think-aloud" from the point they receive the scenario to the point when they reach their final decision. Broadly defined, think-aloud protocols are "self-reported verbal records of thoughts that pass through subjects' minds while performing cognitive tasks" (Johnson, 1993, p. 231). Verbal reports of thought have a long history in psychology, starting with the practice of introspection during the periods of Functionalism and Structuralism (Ericsson \& Simon, 1984). Introspection later came under attack for its unreliable methodology. Watson (1920) argued that introspection was untrustworthy for scientific purposes. However, Watson distinguished between introspection and think-aloud. He later demonstrated that thinking could be made overt, and he argued for the use of think-aloud techniques over the use of introspection.

Following Watson's attack on introspection, the field of psychology largely shifted focus to the study of observable behavior. The use of think-aloud techniques were criticized on three accounts (Ericsson \& Simon, 1984). First, researchers argued that giving verbalizations 
concurrently with one's cognitive processes changed participants' thought processes, and thus the protocols obtained were invalid. This is commonly referred to as the effect of verbalization argument. The second criticism was that participants' may fail to verbalize a large portion of the information that is passing through short-term memory. Therefore, the reports may be limited to the information that the participant decides to report. This is referred to as the incompleteness argument. The final criticism, termed the irrelevant argument, was that the content of verbalizations may reflect an activity that occurs in parallel with the actual thought processes, thus providing limited information about the participants' actual thought processes.

Several studies have addressed the validity of think-aloud protocols by comparing data obtained from think-aloud protocols to written responses to questions regarding decision making strategies, and to information search techniques. Information search techniques generally involve the use of decision matrices, which track what pieces of information participants are accessing and how long they access each piece of information. In a comparison of the thinkaloud technique and a decision matrix technique, Johnson (1993) asked older and younger adults to review information on four apartments and choose one. Half of the participants were instructed to think-aloud as they performed the task, whereas the other half of the participants were given a computer generated decision matrix to access information about each apartment and then indicate their final choice on the computer. Johnson (1993) found that older and younger adults in the think-aloud condition took 30 percent longer to make their decision than those who were using the computer generated decision matrix. However, the type of verbal report condition to which participants were assigned (think-aloud vs. decision matrix) did not affect the final decision that was made. Additionally, age did not affect the average length (number of words) of participants' think-aloud protocols or the amount of time (in seconds) to 
reach a decision. The authors' hypothesized that if the older adult participants had taken significantly longer to reach their decision than the younger adult participants, this would indicate that among older adults, the think-aloud procedure was drawing additional cognitive resources or distracting participants from the decision making task. Taken together, these findings lend support to the validity of the think-aloud procedure for use with both younger and older adults.

Other support for the think-aloud technique can be found in a study by Hoc and Leplat (1983). In a comparison of simultaneous and retrospective verbalization for a computerized sorting task, Hoc and Leplat (1983) asked participants to verbalize their problem solving process both when they were doing the task, and after they completed the task. If participants had difficulty remembering what they had thought during the task, they were shown a record of the steps they had taken to cue recall. Comparing results of the simultaneous and retrospective vocalization conditions, the researchers found no reliable differences in the accuracy of the solution or the number of steps to achieve the solution. They also found that simultaneous verbalization increased the time to make the decision, which is similar to the results of Johnson (1993).

In another demonstration of the validity of the think-aloud procedure, Brinkman (1993) asked undergraduate participants to complete a fault diagnosis for an electrical circuit that was presented to the participants. A fault diagnosis is defined as a solution to why a circuit may be malfunctioning. Brinkman (1993) used three different fault diagnosis tasks and asked participants to report on their problem solving using think aloud reports, retrospective reports, and silent trials. These conditions were counterbalanced across participants. Across all three conditions, the think aloud and retrospective reports did not influence accuracy of the fault 
diagnosis. The time to complete the fault diagnosis was longer in the think-aloud condition than in the other two conditions.

From these and other related studies (e.g., Backlund, Skåner, Montgomery, Bring, \& Strender, 2003; Offredy \& Meerabeau, 2005), one can draw several conclusions. First, thinkaloud reports result in a longer amount of time to complete the problem or make the decision. However, this time difference is likely due to the extra time it takes participants to verbalize their thoughts. Additionally, think-aloud reports do not affect the accuracy of the final solution. Therefore, think-aloud reports have been found to be as valid as retrospective reports of thought process, and as valid as computer generated reports of information accessed via a decision matrix. Some might argue that think-aloud reports lead to richer data than retrospective reports and decision matrix analyses, depending on when the participant is asked to provide a retrospective report. Ericsson and Simon (1993) argued that retrospective reports become less valid as they become farther removed in time from the task at hand, and as the number of trials increases. One additional benefit of think-aloud protocols is that they allow for analysis of how participants are considering and cognitively manipulating each piece of information, which is not available through information obtained from decision matrix studies. Therefore, much of the research on the think-aloud technique supports its use for obtaining a process-oriented account of the decision making task.

\section{Statement of the Problem}

Older adults are potentially making more important and life-threatening medical decisions than any other age group. However, we know little about how older adults make their medical decisions, and how their decision making process differs from younger adults. To examine how older and younger adults might be approaching medical decisions, we can draw on 
both the decision making and problem solving literatures. Although the decision making and problem solving literatures run parallel, the decision making literature has largely ignored an examination of how older adults approach decisions. The problem solving literature, particularly the everyday problem solving literature, has contributed to our understanding of older adult decision making through the examination of the decision making process and the strategies older and younger adults use to solve problems. The everyday problem solving literature has largely examined instrumental and interpersonal tasks among older adults (e.g., Blanchard-Fields et al., 2007; Diehl et al., 1995). These tasks are chosen because they hypothetically occur at a relatively high frequency in older adults' lives, therefore lending themselves to an examination of the role of accumulated wisdom in problem solving, versus the role of primary cognitive abilities.

There are several relevant results from the everyday problem solving literature that provide information on older adult problem solving processes. For instance, everyday problem solving researchers have found that older adults may be using different strategies than younger adults to solve problems (e.g., Blanchard-Fields, Chen, \& Norris, 1997), but may be arriving at equally accurate decisions and equally effective solutions (e.g., Walker et al., 1997). Additionally, researchers have found that older adults engage in other decision processes that might reduce the effectiveness of their problem solving, such as taking less time to make medical decisions than younger adults (Leventhal et al., 1993), seeking less information about treatment alternatives (Meyer et al., 1995), and showing less evidence of engagement of complex comparison and evaluation of the information available to them (Zwahr et al., 1999). Therefore, the results from everyday problem solving studies can help us understand how older adults may be approaching decisions, and how their approach differs from that of younger adults. However, 
everyday problem solving researchers have not studied decisions that occur at a relatively low frequency, but are of paramount importance, such as deciding between medical treatments. Everyday problem solving researchers also have largely ignored the influence of decisional biases on the decisional process of younger and older adults, including an examination of how the presentation of information affects the problem solving process and outcome.

Decisional biases in the area of medical decision making are particularly troubling because the ultimate goal is for patients to make well-informed and unbiased medical decisions. However, there is evidence in the decision making literature that this is not the case (McNeil et al., 1982). In fact, research on biases in decision making has found that the use of decisional biases often precludes well-informed decisions (e.g., Moxey, O'Connell, McGettigan, \& Henry, 2003). One such bias that precludes well-informed and consistent medical decisions is demonstrated through the framing effect.

The framing effect in medical decision making is a robust phenomenon that has been demonstrated with multiple populations and multiple types of medical decisions (e.g., Hux \& Naylor, 1995; Marteau, 1989; McNeil et al., 1982). Many demographic and individual difference variables have been examined in relation to the framing effect and medical decision making (e.g., McKee, 2001; Misselbrook \& Armstrong, 2001; Schuller, 2006; Smith \& Levin, 1996). For instance, Hughes (1993) found that prior personal experience with the medical decision and the experiences of friends and family members (vicarious experience) served as a protective factor. The participants who had prior experience with the medical decision, either through their own experience of the experience of family and/or friends, were less likely to demonstrate the framing effect. 
The variable of age has been examined in a few studies, and these studies support the notion that older adults are potentially more susceptible to the framing effect than younger adults (e.g., Kim et al., 2005; Schuller, 2006). Some researchers suggest that older adults' increased susceptibility to the framing effect is due to the manner in which information is considered (Yates \& Patalano, 1999). Park (1999) proposed that older adults are likely to engage in biased decision making based on age-related decline in cognitive skill and slowed information processing. Therefore, older adults may be more likely to engage in rapid decision making which involves the use of heuristics, and successfully avoids the cognitive demands of an analytic approach to decision making. There is evidence that older adults are engaging in biased decision making, as demonstrated by their susceptibility to the framing effect. All of the studies that have examined the framing effect among older adults have found that older adults exhibited the framing effect in at least one condition (e.g., Kim et al., 2005; McKee, 2001; Schuller, 2006; Woodhead, 2006). However, these studies were inconsistent in their methodology, so it is not clear whether the results for older adults could be replicated given similar methodology. Additionally, not all the older adult participants in these studies demonstrated the framing effect, which leads to the question of why there is variability both between and within data formats.

The foregoing suggests there is inconsistency in results regarding the extent to which older adults are susceptible to the framing effect than younger adults. Many of the cited studies are unpublished, and there are currently no published studies that have examined exactly how older and younger adults consider the presented information in the process of arriving at their final treatment decision, and how their consideration of the information impacts whether they do or do not demonstrate the framing effect. A process oriented approach to examining medical decision making in older and younger adults would permit an examination of the differences in 
how individual older and younger adults engage in the medical decision making process, and an examination of the potential use and influence of heuristics or rules.

\section{Present Study}

The present study attempted to address a number of questions regarding the framing effect in younger and older adults' medical decision making. Many of these questions were addressed through the use of think-aloud protocols. The following questions were addressed: (1a) Will older adults demonstrate the framing effect in either the cumulative or interval probability formats? (1b) Will younger adults demonstrate the framing effect in either the cumulative or interval probability formats? (2) To what extent will the individual difference variables of age, sex, cognitive functioning (measured by the Saint Louis University Mental Status Examination), personal experience with cancer and cancer decision making, vicarious experience with cancer and cancer decision making, and knowledge of the disease predict the framing effect? (3a) What are the differences in how older adults, who do and do not exhibit the framing effect, consider the information that was presented in each data format? (3b) What are the differences in how younger adults, who do and do not exhibit the framing effect, consider the information that is presented in each data format? (4a) What information do participants consider while making their decision in each frame (survival vs. mortality) for the interval probability format, and how does it differ across the age groups? (4b) What information do participants consider while making their decision in each frame (survival vs. mortality) for the cumulative probability format, and how does it differ across the age groups? 


\section{Method}

\section{Participants}

Two groups, younger and older adults, were recruited for participation in the current study. The sample size of 80 participants was estimated to yield sufficient power $(>0.70)$ to detect medium effect sizes for the logistic regression and chi-square analyses. Younger adults enrolled for individual sessions via the West Virginia University SONA system. Older adults completed the study in a quiet location at a senior center. At the end of the study, all participants had the opportunity to enter their names to win one of four $\$ 75$ cash prize drawings. The first group of participants consisted of 40 younger adults ( 25 women and 15 men) recruited from undergraduate psychology classes, who had a mean age of 19.8 years (range $18-24 ; S D=1.5$ ). The majority of the younger adult sample was Caucasian (92.5\%) and single (100\%). Their mean education level was 14.4 years $(S D=1.6)$. Ten percent of the younger adult participants had been diagnosed with a chronic illness. None of the younger adult participants had ever been diagnosed with any type of cancer. Seventy percent of the younger adult participants had discussed the treatment and experience of cancer with someone who had cancer.

The second group of participants consisted of 40 older adults (21 women and 19 men), recruited from local senior centers, who had a mean age of 77.4 years (range $65-89, S D=5.9$ ). The majority of the older adult participants were Caucasian (97.5\%) and either married (35\%) or widowed $(55 \%)$. The older adult participants had a mean education level of 11.4 years $(S D=$ 3.1). A majority of the older adult participants $(82.5 \%)$ had been diagnosed with a chronic illness. A small percentage of the older adults had been diagnosed with cancer at some point in their lives (22.5\%). Forty percent of the older adult participants had discussed the experience and treatment of cancer with someone who had cancer. All participants were asked a series of 
questions regarding their health and their experience with cancer. These responses are presented for each age group in Table 1.

There are several differences to note when examining our sample of younger and older adults. First, the older adults appeared to have more interest in the topic of cancer, as reflected by them reporting higher rates of watching TV and radio programs about cancer. Additionally, they were less likely than younger adults to have discussed the experience of cancer with someone going through it, despite knowing the same number of individuals with cancer. Our older adult sample was less likely than the younger adult sample to have seen their physician in the past four to five months. These differences could reflect our rural sample of older adults who may be less inclined toward preventive health care. Conversely, our younger adult sample may have had required physician visits before being admitted to college, thus making them seem like a group that is more likely to seek timely medical care. One of the most marked differences between our younger and older adult sample was years of education. The younger adults had, on average, three more years of education than the older adults. 
Table 1

Participant Characteristics for Older and Younger Adults

\begin{tabular}{|c|c|c|}
\hline & $\begin{array}{l}\text { Older Adults } \\
(\mathrm{n}=40)\end{array}$ & $\begin{array}{l}\text { Younger Adults } \\
(\mathrm{n}=40)\end{array}$ \\
\hline Watched TV programs about cancer? & $40 \%$ & $87.5 \%$ \\
\hline If so, how influential & $3.2(S D=1.8)$ & $3.2(S D=1.1)$ \\
\hline Listened to radio programs about cancer? & $22.5 \%$ & $12.5 \%$ \\
\hline If so, how influential? & $3.4(S D=1.7)$ & $3.4(S D=1.1)$ \\
\hline Read articles about cancer? & $57.5 \%$ & $87.5 \%$ \\
\hline If so, how influential? & $3.3(S D=1.6)$ & $3.4(S D=1.0)$ \\
\hline \multirow[t]{2}{*}{ Number of weeks since last doctor's appointment } & $27.6(S D=97.6$ & $19.1(S D=24.9$ \\
\hline & Range $=1-624)$ & Range $=1-104)$ \\
\hline \multicolumn{3}{|l|}{ Self-reported health } \\
\hline Poor & $2.5 \%$ & $0 \%$ \\
\hline Fair & $25.0 \%$ & $5 \%$ \\
\hline Average & $7.5 \%$ & $10 \%$ \\
\hline Good & $52.5 \%$ & $40 \%$ \\
\hline Excellent & $12.5 \%$ & $45 \%$ \\
\hline \multirow[t]{2}{*}{ \# of individuals known with cancer } & $4.6(S D=5.7$ & $4.8(S D=5.7$ \\
\hline & Range $=0-30)$ & Range $=0-35)$ \\
\hline
\end{tabular}

Note. Ratings of how influential for TV, radio, and articles ranged from $1=$ not at all important to $5=$ very important. 


\section{Equipment \& Materials}

Equipment. Think-aloud protocols were recorded using a standard audio recorder. The participants were asked to wear lapel microphones while they worked through the think-aloud technique. All interviews were transcribed following the session, and the tapes were stored in a locked file cabinet.

Practice Think-Aloud Problems and Instructions. Participants were provided with detailed instructions on how to complete the think-aloud portion of the study (Appendix A). These instructions were taken from Ericsson and Simon (1984), and emphasized that the participant should keep talking throughout the think-aloud procedure. Prior to asking the participant to practice thinking-aloud, the principle investigator modeled the think-aloud technique using a simple arithmetic problem. Participants were then asked to practice two thinkaloud problems (Appendix B). These problems, taken from Ericsson and Simon (1984), focused on getting the participants to verbalize their thought process while engaging in the task.

Participant Instructions. The participant instructions provided the participant with background information on the two cancer treatment options, surgery and radiation (Appendix C). The instructions also provided information on how most patients feel 6 weeks after receiving either treatment. The 6 week comparison was used to allow participants to compare information on the duration of treatments and the length of recovery. Participants were instructed that this information could be incorporated into their decisional process.

Stimulus Materials. Four hypothetical lung cancer scenarios, taken from McNeil et al. (1982) and McKee (2001) were used as the stimulus materials (Appendix D). The scenarios presented survival and mortality wording for both cumulative probability and interval probability data formats. The scenarios were labeled with different hypothetical hospital names (North, 
South, East, and West Hospital). This was done to encourage participants not to compare the statistics in the four medical scenarios as they progressed through the study. The instructions presented under each scenario instructed the participant to consider only the information presented in the scenario they were reading, in addition to considering personal information and the information presented in the Participant Instructions. Upon completion of the data collection, it was noted that there were typographical errors in the interval probability format, such that individuals were left unaccounted for at the end of the 5-year mark. The potential influence of this error will be further discussed in the limitations section.

Treatment Choice Questionnaire. Following presentation of the stimulus materials, participants were asked to circle which treatment choice they would select for the scenario they just read (Appendix E).

Demographic. All participants completed a demographic questionnaire at the end of the study. The questionnaire asked the participant to provide basic information such as age, gender, years of education, marital status, ethnicity, and current health status (Appendix F). Participants were also asked a series of questions on vicarious experience with cancer, personal experience with cancer, and knowledge of cancer. The knowledge of cancer questions were taken from an online quiz titled, "Lung Cancer: Fact or Fiction?” This website can be located at http://lungdiseases.about.com/library/bllcmythfact1.htm.

Saint Louis University Mental Status Examination (SLUMS). The SLUMS (Appendix G) is a 30 -item cognitive screening instrument that is used to detect the presence of cognitive impairment. Questions assess orientation, memory, attention, and executive functions. As compared to the Mini-Mental State Examination (MMSE), the SLUMS is more sensitive to mild cognitive impairment (Tariq, Tumosa, Chibnall, Perry, \& Morley, 2006). The SLUMS was 
administered to all participants by the examiner to determine whether older and younger adult participants differed with regards to their level of cognitive functioning.

\section{Study Procedure}

Older adult participants completed the study in a quiet location at the senior center from which they were recruited. Younger adults signed up for the study through the West Virginia University SONA system, and completed the study in a laboratory. Younger and older adult participants completed the study one-on-one with the researcher administering all the materials. Participants first received the informed consent form, and any questions regarding the nature of the study were answered. After participants gave consent, the researcher read the participants brief instructions for the think-aloud procedure (Appendix A). The researcher then modeled a think-aloud problem. Participants were asked to complete two think-aloud practice problems (Appendix B). The practice problems were repeated until the participant could talk out loud continuously with no more than a five second break. The participant then read the participant instructions. During the think-aloud procedure, the experimenter prompted the participants to verbalize their thoughts if there was more than five seconds of silence. Their responses were recorded using a standard voice recorder. Participants followed a similar procedure for the remaining three scenarios. Time to decision was recorded for each scenario. Following completion of the think-aloud component, participants completed the demographic questionnaire, were administered the SLUMS examination, and were asked to provide information for the honorarium. Therefore, the participants completed the study materials in the following order: (1) informed consent, (2) instructions for think-aloud procedure, (3) practice think-aloud problems (including researcher modeling), (4) instructions for the medical scenarios (5) scenario \#1 with treatment questionnaire, (6) scenario \#2 with treatment questionnaire, (7) 
scenario \#3 with treatment questionnaire, (8) scenario \#4 with treatment questionnaire, (9) demographic questionnaire, and (10) the SLUMS Examination. The medical scenarios were counter-balanced across participants. To avoid the salience of the framing effect manipulation, the survival and mortality frames of each data format (cumulative probability [CP] and interval probability [IP]) were not juxtaposed. Given this restriction, there were eight possible combinations: (1) CP survival, IP survival, CP mortality, IP mortality, (2) CP survival, IP mortality, CP mortality, IP survival, (3) IP survival, CP survival, IP mortality, CP mortality, (4) IP survival, CP mortality, IP mortality, CP survival, (5) CP mortality, IP mortality, CP survival, IP survival, (6) CP mortality, IP survival, CP survival, IP mortality, (7) IP mortality, CP mortality, IP survival, CP survival, (8) IP mortality, CP survival, IP survival, CP mortality. Qualitative Analysis Procedure

Subsets of interview transcripts were selected for qualitative analysis to answer Research Questions 3a, 3b, 4a, and 4b. For Research Question 3a, all of the transcripts from older adult participants who had demonstrated the framing effect in the expected direction with the interval probability format were used for qualitative analysis $(n=7)$. If a participant's transcript was selected for analysis, transcripts from both the survival and mortality frame were analyzed. This same procedure was used to choose transcripts to analyze for demonstration of the framing effect among older adults in the cumulative probability format $(n=9)$. Transcripts were then chosen randomly without replacement from those older adults that did not demonstrate the framing effect in the interval probability format, and for those who did not demonstrate the framing effect in the cumulative probability format. For the selected transcripts where participants did not demonstrate the framing effect, transcripts were selected randomly without replacement until treatment choice pairings for the survival and mortality frames were equal (e.g., equal number of 
those choosing radiation/radiation and those choosing surgery/surgery), and until the gender distribution matched that of those who did demonstrate the framing effect.

A similar procedure was used to select transcripts for analysis in Research Question $3 \mathrm{~b}$. In the interval probability format, seven younger adults demonstrated the framing effect in the expected direction, therefore, those transcripts were analyzed for both the survival and mortality frame. Random selection without replacement was used to determine those transcripts selected for analysis among younger adults who did not demonstrate the framing effect in the interval probability format. The same procedure was employed to select transcripts from those younger adults who did and did not demonstrate the framing effect in the cumulative probability format. As was done for Research Question 3a, the randomly selected protocols for those younger adults not demonstrating the framing effect were matched in gender and treatment selection to those who did demonstrate the framing effect.

For Research Questions 4a and 4b, ten transcripts were randomly chosen for younger and older adults in both the cumulative and interval probability formats. Transcripts were chosen randomly without replacement until the gender distribution was equal within each set of transcripts (five males, five females). Transcripts were not chosen with regard to who did and did not demonstrate the framing effect, thus number of participants in this analysis who did and not demonstrate the framing effect were not equal (IP younger adults: 1 framer, 9 non-framers; IP older adults: 0 framers, 10 non-framers; CP younger adults: 4 framers, 6 non-framers; $\mathrm{CP}$ older adults: 2 framers, 8 non-framers). Qualitative analyses were carried out on both the survival and mortality frames of the randomly chosen transcripts for Research Questions $4 \mathrm{a}$ and $4 \mathrm{~b}$. The survival and mortality think-aloud transcripts were used for each selected participant to minimize variability that would be introduced by selecting different transcripts for survival frame analyses 
than those selected for mortality frame analyses. That is, using the same participant's survival and mortality transcripts for Research Questions $4 \mathrm{a}$ and $4 \mathrm{~b}$ allowed us to examine only the effect of age on responses, thus ruling out the variability of survival or mortality frame on responses.

Qualitative analyses proceeded in a series of steps. First, the principal investigator selected five undergraduate research assistants (RAs) to assist with data analysis. The RAs were undergraduate psychology students who had no formal experience with conducting qualitative analyses. The project was broadly explained to the RAs and the nature of the think-aloud protocol was also explained. Although no formal training was provided on qualitative analysis, the RAs did practice coding several think-aloud protocols with the principal investigator before beginning analyses.

Following the introduction sessions, the RAs began analyses on the project data. With the principal investigator, the RAs read the selected transcript in its entirety. Each person in the coding group then wrote down descriptive subcategories that they thought best captured the content. Everyone read their subcategories out loud to the coding group and discussed why they had chosen the subcategories. Final subcategories were decided on by agreement between the coding group. These subcategories were then defined and retained to use for future transcripts. Subcategories were coded as either present or absent, and not according to how many times each subcategory appeared in the transcripts. The RAs were blind to the different conditions that were represented in each transcript (e.g., age, frame, interval/cumulative probability format). Following completion of assigning subcategories to each selected transcript, the principal investigator then sorted the subcategories for each transcript into tables for the appropriate research question (e.g, older adults who did not demonstrate the framing effect), and created 
overarching categories that were intended to capture multiple subcategories. Definitions were also created by the principal investigator for the overarching categories.

Five overarching categories were created based on the nature of the individual subcategories. These categories were then applied to each research question. The five overarching categories were: personal decision making factors, comparative decision making factors, one-sided decision making factors, treatment specific decision making factors, and decision based primarily on a single factor. Appendix $\mathrm{H}$ lists the five overarching categories with the individual subcategories listed below. Appendix I lists each individual subcategory with an example of the subcategory that was taken directly from participants' interview transcripts. While conducting the qualitative analyses, the research team also noted whether the participant referenced any of the presented data in their transcripts.

The word choice used for creation of the 30 individual subcategories is important, as it reflects the degree of weight that was given to different pieces of information. Individual subcategories that begin with "comparison of" were assigned to instances where participants were comparing numerical aspects of each treatment, either with a direct comparison of the presented data, or a qualitative comparison of the presented data (e.g., more than or less than). The subcategories that begin with, "weighed" indicate that the participant was considering nonnumerical aspects of each treatment. Subcategories that begin with, "reference to" indicate that the participant mentioned a particular piece of information, but did not provide any information as to how important the information was to his or her decision. Finally, subcategories that begin with, "consideration of" reflect that participant mentioned one side of the information as being important in their choice, but there was no comparison of this piece of information across treatment options, and no indication of whether the information influenced their final decision. 
Following creating of the overarching categories, two new undergraduate research assistants (who did not participate in the first set of coding) were asked to sort the 30 subcategories into the appropriate overarching categories. They were used as a "feedback" group to help with clarifying the definitions of the subcategories and overarching categories. Due to this, these two raters were given 10 subcategories at a time, and were then asked to provide feedback before proceeding to the next set of 10 subcategories. Kappa was calculated for each of the three sets of 10 subcategories, and for the overall 30 subcategories. After refining the subcategories based on feedback from these two raters, two new raters were asked to sort the 30 subcategories into the five overarching categories without any feedback from the principal investigator. Kappa was also calculated for this set of raters based on all 30 subcategories. The materials that were presented to the two independent raters are presented in Appendix J.

Results

\section{Statistical Analyses}

Alpha level was set at 0.05 for all analyses. Analyses were conducted in SPSS (version 15). Chi-square tests were conducted to answer Research Questions 1a and 1b, which examined the presence of a framing effect among younger and older adults with the interval and cumulative probability outcome formats. Logistic regression was used to analyze Research Question 2, which examined predictors of the framing effect. The forced entry method was used for all logistic regression analyses.

\section{Initial Analyses}

Older and younger adult participants were compared on several variables to determine statistically significant differences. For the cancer knowledge quiz, older adult participants scored significantly lower $(M=4.70, S D=1.18)$ than younger adult participants $(M=5.50, S D=$ 
$1.20), t(78)=-3.01, p<0.01$. For the other three variables of interest, Levene's Test for Equality of Variance was significant, therefore violating the assumption of homogeneity of variances. Therefore, t-tests were conducted using Welch's t-test, which is used by SPSS when the homogeneity of variance assumption is violated. Younger adults scored significantly higher ( $M$ $=25.48, S D=2.56)$ than older adults $(M=21.25, S D=5.43)$ on the SLUMS, $t(55.55)=-4.45, p$ $<0.001$, Levene's $\mathrm{F}(1,78)=16.58, p=0.00$. Younger adults also reported significantly more years of education $(M=14.41, S D=1.58)$ than older adults $(M=11.44, S D=3.14), t(57.61)=$ $5.36, p<0.001$, Levene's $\mathrm{F}(1,78)=8.84, p=0.00$. Younger adults rated their health as significantly better $(M=4.25, S D=0.84)$ than older adults $(M=3.48, S D=1.09), t(73.36)=-$ 3.57, $p<0.001$, Levene's $\mathrm{F}(1,78)=5.04, p=0.03$, and older adults reported significantly more chronic illnesses $(M=1.20, S D=0.88)$ than younger adults $(M=0.10, S D=0.30), t(48.11)=$ $7.45, p<0.001$, Levene's $\mathrm{F}(1,78)=20.23, p=0.00$.

\section{Research Questions}

Research Question 1a was proposed to examine the presence of a framing effect among older adults in the cumulative and interval probability outcome formats. A 2 (surgery or radiation in the mortality frame) $\mathrm{x} 2$ (surgery or radiation in the survival frame) chi-square test was used for each format. Interpretation of the chi-square results was done in two steps. First, statistical significance of the chi-square value was examined. If the chi-square value was not significant then the proportion of responses was not significantly different than expected and analyses stopped at this step. If the chi-square value was significant, the distribution of responses within the chi-square table was examined to determine whether the significance was indicative of a framing effect. Thus, given the non-directional nature of the chi-square test, a significant chi-square value indicates that the significant differences could lie in either direction 
(top left to bottom right diagonal vs. top right to bottom left diagonal). For example, if most responses were distributed on the top left to bottom right diagonal, this would indicate that a significant proportion of participants chose the same treatment across the survival and mortality condition. If, on the other hand, most responses were distributed on the top right to bottom left diagonal, this would indicate that a significant proportion of participants changed their response from either surgery in the survival frame to radiation in the mortality frame, or from surgery in the mortality frame to radiation in the survival frame. The results for older adults with both the cumulative and interval probability format are presented in Table 2.

Table 2

Framing Effect Test among Older Adults

Mortality Frame

Interval Probability** Cumulative Probability*

$$
(\mathrm{n}=40) \quad(\mathrm{n}=40)
$$

\begin{tabular}{ccrrrr}
\hline & & Surgery & Radiation & Surgery & \multicolumn{1}{c}{ Radiation } \\
Survival & Surgery & $16(40 \%)$ & $7(17.5 \%)$ & $12(30 \%)$ & $9(22.5 \%)$ \\
Frame & Radiation & $3(7.5 \%)$ & $14(35 \%)$ & $2(5 \%)$ & $17(42.5 \%)$ \\
\hline$* * p<.001, * p<.01$. & & & &
\end{tabular}

The chi-square value was significant for both the interval probability $\left(\chi^{2}(1)=10.57, p<\right.$ $.001)$ and cumulative probability $\left(\chi^{2}(1)=9.53, p<.01\right)$ outcome formats. An examination of the contingency table revealed that the majority of responses were distributed in the "no framing" direction. For the interval probability format, 40 percent of participants chose surgery in both frames, and 35 percent of participants chose radiation in both frames. For the cumulative probability format, 30 percent of participants chose surgery in both frames and 42.5 percent of 
participants chose radiation in both frames. Therefore, a larger proportion of responses were distributed along the top left to bottom right diagonal, indicating that a statistically significant proportion of the older adult participants chose the same treatment choice regardless of frame. Thus, these analyses indicated that older adults did not demonstrate a framing effect in either outcome format. The selection among participants of each treatment choice across the different formats was relatively equal. That is, with the interval probability format, 16 older adult participants chose surgery in both frames, compared to 14 who chose radiation in both frames. A similar pattern was observed for the cumulative probability format, where responses were similar between participants who chose surgery in both formats $(n=12)$ and those who chose radiation in both formats $(n=17)$, indicating that participants did not tend to favor one treatment over the other. A statistically significant phi value of $0.51(p<.001)$ was obtained for the interval probability format, which corresponds to a small to medium effect size. A statistically significant phi value of $0.49(p<.01)$ was obtained for the cumulative probability format, which corresponds to a small effect size.

Research Question 1b was proposed to examine the presence of a framing effect among younger adults in the cumulative and interval probability outcome formats. A 2 (surgery or radiation in the mortality frame) $\mathrm{x} 2$ (surgery or radiation in the survival frame) chi-square test was used for each format. Results are presented in Table 3. 
Table 3

Framing Effect Test among Younger Adults

\begin{tabular}{cccccc}
\hline & & \multicolumn{3}{c}{ Mortality Frame } \\
\cline { 3 - 6 } & & \multicolumn{2}{c}{$(\mathrm{n}=40)$} & \multicolumn{2}{c}{ Cumulative Probability } \\
& & Surgery & Radiation & Surgery & Radiation \\
\hline \multirow{2}{*}{ Survival } & Surgery & $22(55 \%)$ & $7(17.5 \%)$ & $10(25 \%)$ & $16(40 \%)$ \\
Frame & Radiation & $1(2.5 \%)$ & $10(25 \%)$ & $2(5 \%)$ & $12(30 \%)$ \\
\hline$* * p<.001$. & & & & &
\end{tabular}

For the interval probability format among younger adults, a statistically significant chisquare value was obtained, $\chi^{2}(1)=14.55, p<.001$. An examination of the contingency table revealed that the distribution of responses was significant in the "no framing" direction. That is, 55 percent of the participants chose surgery in both the mortality and survival frames, and 25 percent chose radiation in both frame. A statistically significant phi value of $0.61(p<.001)$ corresponds to a medium effect size for this format. The obtained chi-square value for the cumulative probability condition was not significant $\left(\chi^{2}(1)=2.53, p=0.11\right)$, indicating that the proportion of responses in each cell was not significantly different than expected.

Two separate logistic regression analyses were conducted to examine predictors of the framing effect in the entire sample of younger and older adults. The dependent variable for the first analysis was whether participants demonstrated the framing effect in the interval probability format. In the second analysis, the dependent variable was whether participants demonstrated the framing effect in the cumulative probability format. These two variables were created by determining whether each participant reversed his or her decision in any direction across the 
survival and mortality frames of the interval probability format. If a reversal was present, they were assigned a code of ' 1 ' for demonstrating a framing effect in the interval probability format. A code of '0' was assigned for no choice reversal. A similar procedure was used to determine demonstration of a framing effect in the cumulative probability format. Thus, the dependent variables in the two logistic regression analyses were either demonstration of framing in the interval probability format or demonstration of framing in the cumulative probability format. In the cumulative probability format, $29(36.3 \%)$ of the participants demonstrated the framing effect and $51(64.7 \%)$ did not. For the interval probability format, $18(22.5 \%)$ of the individuals demonstrated the framing effect and $62(77.5 \%)$ did not. The predictor variables for both logistic regression analyses included: age, sex, score on the SLUMS, score on the knowledge questionnaire, personal experience with cancer, and vicarious experience with cancer. Age was coded into two discrete values $(1=$ older adults, 2 =younger adults $)$, as was sex $(1=$ male, $2=$ female). With the exception of the dichotomously coded age and sex variables, all predictor variables were examined for normality prior to running the analyses. Skewness and kurtosis values were all within acceptable ranges.

The score on the SLUMS was obtained according to the scoring criteria outlined on the measure. The range of scores for the SLUMS among both younger and older adults was 8 to 30 , with a mean score of $23.36(S D=4.72)$. The knowledge score was obtained by summing correct responses to the nine questions, with each correct response assigned one point. Scores ranged from 3 to 8 with a mean of $5.10(S D=1.25)$.

The personal experience with cancer variable was created by assigning points for responses to the following four questions: "Have you ever been diagnosed with cancer?" and "If so, please indicate the type of cancer, the treatment you received, and when you were 
diagnosed." Points were assigned as follows: one point if the participant had been diagnosed with cancer, one point if the type of cancer was lung, one point if the treatment received was either surgery or radiation, and one point if the participant was diagnosed within the last five years. Scores on this variable ranged from 0 to 3 , with a mean of $0.23(S D=0.66)$. To create the vicarious experience variable, points were assigned for the following questions: "How many individuals do you know that have ever been diagnosed with any type of cancer?" and "If you knew someone who was ever diagnosed with cancer, please rate how involved you were in this person's life during their experience with cancer." Participants received one point for each five individuals they knew with cancer (e.g., 15 individuals $=3$ points). Points on the second question were taken from the Likert-type response scale of 1 (not at all involved) to 5 (very involved). For the entire sample, scores on the vicarious experience variable ranged from 1 to 12 with a mean of $3.52(S D=2.57)$.

The logistic regression model that examined predictors of a framing effect with the interval probability format was not supported, $\chi^{2}(6, N=80)=7.02, p=0.32$. The logistic regression model that examined predictors of a framing effect with the cumulative probability format was also not supported, $\chi^{2}(6, N=80)=10.67, p=0.09$. Because this analysis included both age groups of participants, we decided to further explore the influence of the predictor variables for the cumulative probability format by splitting the analysis into two separate age groups. Therefore, two logistic regression analyses were conducted separately for the younger and older adult samples, using the following predictor variables: sex, knowledge score, SLUMS score, personal experience with cancer variable, and vicarious experience with cancer variable. Among the older adult participants, the logistic regression model was not supported, $\chi^{2}(5, N=$ 
$40)=2.80, p=0.73$. Among the younger adult participants, the overall model was supported, $\chi^{2}$ $(5, N=40)=12.26, p<0.05$. The results are presented in Table 4 .

Table 4

Logistic Regression Results for Younger Adults with Cumulative Probability Format

\begin{tabular}{lcccc}
\hline Variable & $B$ & $S E$ & $\begin{array}{c}\text { Odds Ratio } \\
(95 \% \mathrm{CI})\end{array}$ & Wald Statistic \\
& & & & \\
\hline Cumulative Probability $(\mathrm{n}=40)$ & & & $0.48(0.09-2.51)$ & 0.75 \\
Sex & -0.73 & 0.84 & 0.45 & 0.34 \\
SLUMS Score & -0.10 & 0.18 & $0.90(0.64-1.28)$ & $4.85^{*}$ \\
Knowledge Score & 0.96 & 0.43 & $2.60(1.11-6.10)$ & 1.08 \\
Vicarious Experience & -0.10 & 0.10 & $0.90(0.75-1.09)$ & 0.42 \\
Personal Experience & -0.91 & 1.40 & $0.40(0.03-6.25)$ & \\
\hline$* p<.05$ & & & &
\end{tabular}

The results for the younger adult participants with the cumulative probability format indicate that as knowledge score increased, the probability of demonstrating the framing effect also increased $(\mathrm{O} . \mathrm{R} .=2.60, p<.05)$. Assessment of the fit of the regression model revealed a non-significant Hosmer \& Lemeshow's test, $\left(\chi^{2}(8, N=40)=9.98, p=0.27\right)$, indicating that the model did not differ significantly from the observed data. A measure of effect size, Negelkerke's $R^{2}$, revealed a medium effect size for the model $\left(R^{2}=0.35\right)$. An assessment of multicollinearity of the predictor variables revealed no tolerance values less than 0.10 and no VIF values greater than 10 . 


\section{Qualitative Analyses}

Interrater reliability was calculated for sorting the 30 subcategories into their respective overarching categories. For the first pair of raters, kappa was 0.36 for the first ten subcategories, 0.87 for the second set of ten subcategories, and 0.87 for the third set of ten subcategories. Overall kappa for the first pair of raters was 0.70 . The first pair of raters provided feedback on the clarity of the subcategories between each set of ten subcategories, and those modifications were then used for the second set of ten subcategories. Further refinements were made after completing the second set of ten subcategories, and those changes were then used for the final set of ten subcategories. The second pair of raters were given all 30 subcategories and worked through the materials without clarification from the principal investigator. Kappa for the second pair of raters was 0.78 .

Due to the large size of the tables that present the results of the qualitative analyses, the main results are summarized in this section, and the reader is referred to the tables in Appendices $\mathrm{K}$ to $\mathrm{N}$.

Research Question 3a examined the differences in how older adults, who did and did not demonstrate the framing effect, considered the presented information in each data format. Several interesting results were obtained, which will be reviewed according to probability format.

Interval Probability Format. In the survival framed scenario with the interval probability format, the majority of the older adults who were selected for qualitative analysis and demonstrated the framing effect $(\mathrm{n}=7)$ discussed comparative decision making factors $(\mathrm{n}=5)$ and personal decision making factors $(\mathrm{n}=5)$, whereas those that did not demonstrate the framing effect $(n=7)$ discussed personal decision making factors $(n=6)$ and based their decision 
primarily on a single factor $(n=4)$. Additionally, those that demonstrated the framing effect were more likely to reference data that were presented in the scenario $(n=4)$. In the mortality framed scenario with the interval probability format, similar results were obtained. Those older adults that demonstrated the framing effect $(n=7)$ discussed comparative $(n=5)$ and one-sided decision making factors $(\mathrm{n}=5)$, whereas those that did not demonstrate the framing effect $(\mathrm{n}=$ 7) discussed personal decision making factors $(n=6)$, and based their decision on a single factor $(n=5)$. Similar to results obtained for the survival framed scenario, older adults that demonstrated the framing effect in this format were more likely to reference the presented data $(n=6)$.

Cumulative Probability Format. In the survival framed scenario with the cumulative probability format, older adults that demonstrated the framing effect $(n=9)$ discussed comparative $(n=5)$ and one-sided decision making factors $(n=4)$, whereas those not demonstrating the framing effect $(n=9)$ discussed one-sided decision making factors $(n=6)$ and based their decision primarily on a single factor $(n=6)$. For the mortality framed scenario with the cumulative probability format, differences between framers and non-framers were less clear. Those older adults that demonstrated the framing effect $(n=9)$ discussed one-sided decision making factors $(n=7)$, and based their decision primarily on a single factor $(n=6)$. Those that did not demonstrate the framing effect $(n=9)$ discussed one-sided $(n=6)$ and treatment specific decision making factors $(\mathrm{n}=5)$.

Research Question 3b examined the differences in how younger adults, who did and did not demonstrate the framing effect, considered the presented information in each data format. Results will be reviewed according to probability format. 
Interval Probability Format. In the survival framed scenario with the interval probability format, both groups (framers, $\mathrm{n}=7$; non-framers, $\mathrm{n}=7$ ) were likely to discuss comparative decision making factors (framers, $n=13$; non-framers, $n=14$ ), followed by one-sided factors (framers, $\mathrm{n}=3$; non-framers, $\mathrm{n}=4$ ). Those that demonstrated the framing effect did not discuss treatment specific decision making factors. Those that did not demonstrate framing generally considered more factors in multiple overarching categories than those who did demonstrate the framing effect. In the mortality framed scenario with the interval probability format, those that demonstrated a framing effect $(n=7)$ based their decisions primarily on a single factor $(n=8)$, and often used comparative decision making factors $(n=7)$. Those not demonstrating the framing effect $(n=7)$ most often discussed comparative decision making factors $(n=9)$ and based their decisions primarily on a single factor $(n=6)$.

Cumulative Probability Format. Slightly different results were obtained when younger adults were presented with the cumulative probability format. In the survival framed scenario with the cumulative probability format, a majority of younger adults that demonstrated the framing effect $(n=16)$ discussed comparative $(n=33)$ and one-sided decision making factors $(n$ $=10)$, whereas those that did not demonstrate the framing effect $(n=14)$ frequently discussed comparative decision making factors $(n=18)$, and based their decision primarily on a single factor $(n=9)$. In the mortality framed scenario with the cumulative probability format, similar results were obtained. Comparative $(n=18)$ and one-sided decision making factors $(n=7)$ were frequently discussed by those younger adults demonstrating the framing effect $(n=16)$. Those not demonstrating the framing effect $(n=14)$ discussed comparative decision making factors $(n$ $=13)$, and based their decision primarily on a single factor $(\mathrm{n}=12)$. 
Research Question 4a examined what information participants considered while making their decision in each frame (survival vs. mortality) for the interval probability format, and how that differed across the two age groups. In the survival framed scenario of the interval probability format, younger adults sampled for qualitative analysis $(\mathrm{n}=10)$ frequently discussed comparative $(n=16)$ and one-sided decision making factors $(n=10)$, reflecting a focus on the presented data and the side effects and/or process of one of the treatments. Older adults sampled for qualitative analysis $(n=10)$ frequently discussed one-sided $(n=8)$ and personal decision making factors $(n=7)$. Younger adults did not discuss any personal decision making factors in this scenario. Younger adults were also more likely than older adults to reference data presented in the scenario (younger, $n=9$; older, $n=3$ ). In the mortality framed scenario with the interval probability format, younger adults $(n=10)$ again frequently discussed comparative decision making factors $(n=11)$, and based their decision primarily on a single factor $(n=6)$. Older adults $(n=10)$ frequently based their decision on a single factor $(n=8)$, and frequently considered personal decision making factors $(n=7)$. In the mortality framed scenario with the interval probability format, younger adults were also more likely than older adults to reference the data presented in the scenario (younger, $n=9$; older, $n=5$ ).

Research Question 4b examined what information participants considered while making their decision in each frame (survival vs. mortality) for the cumulative probability format, and how that differed across the two age groups. In the survival framed scenario with the cumulative probability format, younger adults $(\mathrm{n}=10)$ frequently discussed comparative decision making factors $(n=13)$, and based their decision on a single factor $(n=8)$. Older adults $(n=10)$ equally discussed multiple factors, including one-sided $(n=4)$, treatment specific $(n=4)$, and personal decision making factors $(n=4)$, and also often based their decision on a single factor $(n=4)$. 
Younger adults were also more likely than older adults to reference the data presented in the scenario (younger, $\mathrm{n}=8$; older, $\mathrm{n}=2$ ).

In the mortality framed scenario with the cumulative probability format, younger adults $(n=10)$ often discussed comparative $(n=9)$ and one-sided decision making factors $(n=4)$. A similar pattern was obtained for the older adult participants $(n=10)$, who often discussed onesided $(n=8)$ and comparative decision making factors $(n=7)$. Younger adults were also more likely than older adults to reference the data presented in the scenario (younger, $\mathrm{n}=9$; older, $\mathrm{n}=$ 5).

\section{Exploratory Questions}

In order to ensure that the survival and mortality frames of each outcome format were not juxtaposed, eight different order combinations were distributed sequentially among participants. Two separate logistic regression analyses were conducted to examine presence of an order effect in each outcome format. Order was entered as a categorical independent variable in a logistic regression analysis. The dependent variables were the demonstration of the framing effect variables created for each scenario (interval probabilities and cumulative probabilities) for the logistic regression analyses in Research Question 2. For the interval probability format, the overall model was not supported, $\chi^{2}(7, N=80)=8.89, p=0.26$. The overall model was also not supported for the cumulative probability format, $\chi^{2}(7, N=80)=7.74, p=0.36$. These results indicate that the order in which participants received the medical scenarios did not significantly affect whether they demonstrated the framing effect in either outcome format.

Additional order effects may have been present in the qualitative component of the study. That is, participants may have had their "best" think-aloud data when they received a certain scenario first, as compared to other participants who received the same scenario second, third, or 
fourth. To determine the "quality" of think-aloud data when different medical scenarios were received first versus second, third, or fourth, time to decision in seconds was recorded, and the number of words in each participant's think-aloud protocol for each medical scenario was also recorded. If there was a "qualitative" order effect, we might expect that those who received a certain scenario first took longer to make the decision and had a greater number of words in their think-aloud protocols, as compared to those who did not receive the scenario first. To address this, we examined systematic differences among the different orders of the four medical scenarios. The results for time to decision and word count are presented in Table 5.

Table 5

Time to Decision and Word Count for Qualitative Order Effects

Order of Presentation

\begin{tabular}{ccccccccc} 
& \multicolumn{3}{c}{ First } & \multicolumn{2}{c}{ Second } & \multicolumn{2}{c}{ Third } & \multicolumn{2}{c}{ Fourth } \\
\hline Scenario & Time & Words & Time & Words & Time & Words & Time & Words \\
& $(S D)$ & $(S D)$ & $(S D)$ & $(S D)$ & $(S D)$ & $(S D)$ & $(S D)$ & $(S D)$ \\
\hline CPS & 91.85 & 129.30 & 62.55 & 106.65 & 53.75 & 114.80 & 61.05 & 121.45 \\
& $(51.78)$ & $(33.64)$ & $(33.39)$ & $(60.09)$ & $(21.57)$ & $(118.38)$ & $(47.72)$ & $(100.30)$ \\
CPM & 104.00 & 176.75 & 66.10 & 113.85 & 72.30 & 113.95 & 62.85 & 103.00 \\
& $(63.57)$ & $(106.06)$ & $(47.06)$ & $(86.76)$ & $(32.07)$ & $(71.84)$ & $(40.55)$ & $(63.89)$ \\
IPS & 104.80 & 183.40 & 85.50 & 145.55 & 71.30 & 128.75 & 83.55 & 134.15 \\
& $(54.43)$ & $(97.97)$ & $(41.53)$ & $(75.69)$ & $(42.79)$ & $(92.57)$ & $(39.98)$ & $(52.09)$ \\
IPM & 111.80 & 207.25 & 79.95 & 112.05 & 62.35 & 91.25 & 82.05 & 153.20 \\
& $(60.00)$ & $(93.56)$ & $(27.72)$ & $(42.81)$ & $(39.76)$ & $(90.27)$ & $(70.53)$ & $(136.29)$
\end{tabular}


Several differences emerged upon examination of Table 5. Participants did take a longer amount of time for the first scenario received in the think-aloud portion of the study, as can be seen by comparing the first column (time in seconds for each of the four scenarios presented first), to values for time to decision located in the third, fifth, and seventh columns. However, time to decision did not systematically decrease across orders. If participants did provide the best quality think-aloud data for the scenario they received first, one might expect time to decision to decrease systematically across order of presentation, which did not occur. The order differences were less pronounced when examining the mean word count in the think-aloud protocols. That is, participants generally took longer to make their decision when a scenario was presented first versus second, third, or fourth, but there were no systematic differences in the number of words in each protocol. It is likely that the differences obtained with the time to decision variable does not pose a large threat to the validity of the think-aloud protocols, given that the differences were not systematic between the different order presentations within each medical scenario.

Time to decision and word count were also examined for statistically significant age differences. Independent samples t-tests, grouped by age, were conducted to examine younger and older adults' average time to decision and average word count for each of the four medical scenarios. Normality of the variables was checked prior to running the analyses. Skewness and kurtosis of each of the variables used in the comparisons were within acceptable limits. For all comparisons, Levene's test for equality of variance was not significant, which satisfied the assumption that the two groups for each comparison have approximately equal variances on the dependent variable. One statistically significant difference was observed between older $(M=$ 
$116.18, S D=86.88)$ and younger adults' $(M=165.70, S D=115.74)$ word count for the mortality framed scenario with the interval probability format, $t(78)=-2.16, p<.05$.

The data were also examined to determine the number of errors made by each participant for each data format. An error was marked if the participant incorrectly cited the data or made an incorrect comparison of the presented data. Number of errors was computed by reading each participant's transcript and comparing the information discussed in the think-aloud portion of the study to the data presented in each data format. If the participant did not reference any data from the scenario they were responding to, they were assigned a code of -99 for that scenario. Error rates were compared between younger and older adult participants and between those participants that demonstrated the framing effect and those that did not. For each group, the number that referenced the data presented in the scenario was also added to the table. The data for average number of errors for younger and older adults are presented in Table 6.

Table 6

Average Number of Errors for Younger and Older Adult Participants

\begin{tabular}{lcccc}
\hline & $\mathrm{CPS}^{\dagger}$ & $\mathrm{CPM}^{\dagger}$ & $\mathrm{IPS}^{+}$ & $\mathrm{IPM}^{\dagger}$ \\
\hline Older Adults $(\mathrm{n}=40)$ & & & & \\
Average Number of Errors & $0.16(0.37)$ & $0.43(0.59)$ & $0.42(0.51)$ & $0.10(0.31)$ \\
Percentage who referenced data & $47.5 \%$ & $57.5 \%$ & $47.5 \%$ & $50.0 \%$ \\
Younger Adults $(\mathrm{n}=40)$ & & & & \\
Average Number of Errors & $0.21(0.48)$ & $0.45(0.81)$ & $0.10(0.30)$ & $0.22(0.48)$ \\
Percentage who referenced data & $92.5 \%$ & $100.0 \%$ & $100.0 \%$ & $92.5 \%$ \\
\end{tabular}

Note. *Error differences: $t(24.33)=2.55, p<.05$, Levene's test: $\mathrm{F}(1,57)=28.70, p=0.00$;

t Percentage differences: $p<.001$ for all comparisons 
Independent samples t-tests were conducted to compare average error rates between younger and older adult participants. Although there were few errors made overall, it is interesting to note that older adult participants made significantly more errors than younger adults on only one data format, the survival framed scenario with the interval probability format, $t(24.33)=2.55, p<.05$. Levene's test for equality of variance was significant for this comparison, $\mathrm{F}(1,57)=28.70, p=$ 0.00 , thus the t-test data that are presented do not assume equal variances. Chi-square tests were used to compare the proportion of older adults referencing the data to the proportion of younger adults referencing the data. All four comparisons were significant at the $p<.001$ level (CPS: $\chi^{2}$ $(1, N=80)=19.27, p<0.001 ;$ CPM: $\chi^{2}(1, N=80)=21.59, p<0.001$; IPS: $\chi^{2}(1, N=80)=$ 28.48, $p<0.001$; IPM: $\left.\chi^{2}(1, N=80)=17.64, p<0.001\right)$. The same results are presented in Table 7 to compare error rates and number who referenced the data in the scenario for those who demonstrated the framing effect and for those that did not.

Table 7

Average Number of Errors for Those Demonstrating the Framing Effect vs. Those Not

$\mathrm{CPS}^{\dagger} \mathrm{CPM}^{* \dagger} \quad \mathrm{IPS}^{\dagger} \quad \mathrm{IPM}$

\section{Framing}

Average Number of Errors

$0.15(0.36)$

$0.86(0.90)$

$0.29(0.47)$

$0.07(0.26)$

Percentage who referenced data

$93.1 \%$

$96.6 \%$

$94.4 \%$

$83.3 \%$

No Framing

Average Number of Errors

Percentage who referenced data
$0.24(0.51)$

$0.11(0.32)$

$0.17(0.38)$

$0.21(0.47)$

$56.9 \% \quad 68.6 \%$
$67.7 \%$

$67.7 \%$

Note. *Error differences: $t(32.68)=-4.20, p<.001$, Levene's test: $\mathrm{F}(1,61)=31.91, p=0.00$;

+ Percentage differences: $p<.05$ for all comparisons 
Independent sample t-tests were conducted to compare average error rates among those demonstrating the framing effect and those not. Separate t-tests were conducted to compare those that demonstrated the framing effect in the cumulative probability format and those that demonstrated it in the interval probability format. One comparison was statistically significant, indicating that those that demonstrated the framing effect in the cumulative probability format made significantly more errors in the mortality frame than those who did not demonstrate the framing effect, $t(32.68)=-4.20, p<.001$. Levene's test for equality of variance was significant for this comparison, $\mathrm{F}(1,61)=31.91, p=0.00$, thus the $\mathrm{t}$-test data that are presented do not assume equal variances. Chi-square tests were used to compare the proportion of framers referencing the data to the proportion of non-framers referencing the data. Three of the four comparisons were significant at the $p<.05$ level (CPS: $\chi^{2}(1, N=80)=11.56, p<0.001$; CPM: $\chi^{2}(1, N=80)=8.62, p<0.01 ;$ IPS: $\chi^{2}(1, N=80)=5.14, p<0.05 ;$ IPM: $\chi^{2}(1, N=80)=1.66, p$ $=0.20)$.

For the transcripts that were selected for qualitative analyses, the number of subcategories assigned to each transcript was recorded. These data were examined for each cell in the qualitative analyses tables to determine the average, minimum, and maximum number of subcategories assigned to each individual to determine how many pieces of information were being considered by the participants whose transcripts were selected for qualitative analyses. For Research Question 3a, the following means were obtained: IPS: $M=3.64, S D=2.17$, Range $=1$ -9; IPM: $M=3.14, S D=1.41$, Range $=1-6$; CPS: $M=3.06, S D=1.21$, Range $=1-5$; CPM: $M=3.11, S D=1.84$, Range $=1-8$. For Research Question 3b, the following means were obtained: IPS: $M=3.36, S D=1.21$, Range $=1-6$; IPM: $M=2.86, S D=1.03$, Range $=2-5$; CPS: $M=3.63, S D=1.52$, Range $=2-8 ; \mathrm{CPM}: M=2.83, S D=1.29$, Range $=1-5$. For 
Research Question 4a, the following means were obtained: IPS: $M=3.80, S D=1.44$, Range $=2$ - 6; IPM: $M=3.60, S D=1.60$, Range $=2-8$. Finally, for Research Question 4b, the following means were obtained: CPS: $M=2.95, S D=0.76$, Range $=2-5 ; \mathrm{CPM}: M=2.95, S D=1.54$, Range $=1-6$

The next exploratory research question aimed to examine differences on demographic variables between those younger and older adults who did and did not demonstrate the framing effect. Independent sample t-tests were conducted separately for both age groups and for both outcome formats. That is, differences between older adults who did and did not demonstrate the framing effect in the cumulative probability format were examined separately from those who did and did not demonstrate the framing effect in the interval probability format. Similar analyses were conducted for younger adults. For both age groups, the following independent variables were entered: age, education, self-rated health, number of individuals known with cancer, score on the knowledge quiz, and score on the SLUMS.

For older adult participants in the cumulative probability format, no significant differences were obtained on the test variables. However, it was noted that older adults who demonstrated the framing effect in this format knew, on average, fewer individuals who had cancer $(M=3.36, S D=2.16)$, as compared to those who did not demonstrate the framing effect $(M=5.03, S D=6.51)$. Additionally, the number of individuals known with cancer for those demonstrating the framing effect ranged from 1 to 7 , whereas for those who did not demonstrate the framing effect, the number of individuals known with cancer ranged from 0 to 30.

For older adult participants in the interval probability format, no significant differences were obtained. However, as seen in the cumulative probability format, older adults who demonstrated the framing effect in this format knew, on average, fewer individuals who had 
cancer $(M=2.40, S D=1.43$, Range $=0-5)$ than those who did not demonstrate the framing effect $(M=5.30, S D=6.35$, Range $=0-30)$.

For younger adult participants in the cumulative probability format, one significant difference was obtained. Those who demonstrated the framing effect in the cumulative probability format scored significantly higher on the knowledge test $(M=6.06, S D=0.87)$ than those who did not demonstrate the framing effect $(M=5.05, S D=1.25 ; t(37.15)=-2.99, p<$ $.01)$. Levene's test for equality of variance was significant for this comparison, $\mathrm{F}(1,38)=4.48, p$ $=0.04$, thus the t-test data that are presented do not assume equal variances.

For younger adult participants in the interval probability format, one significant difference was obtained. Those who demonstrated the framing effect in the interval probability format had significantly more years of education $(M=15.38, S D=2.13)$ than those not demonstrating the framing effect $(M=14.17, S D=1.35) ; t(38)=-1.99, p<.05$.

The final exploratory research question examined what the most influential pieces of information were in the decisional process for younger and older adults. Following completion of the decision making scenarios, all participants were asked, "Please make a list of the major factors that influenced your decisions for the four medical scenarios you just read." Because this question was asked of the participants following completion of all four scenarios, participants' responses to this question were classified into the "demonstrated framing effect" category if they demonstrated a framing effect in either the cumulative or interval probability format. A participant who did not demonstrate a framing effect in either format was placed in the "did not demonstrate a framing effect" category for this question, thereby not differentiating between those who demonstrated a framing effect with one data format but not the other. The principal investigator created tables to examine responses to this question by age (young vs. old) and by 
demonstration of the framing effect (older adults demonstrating the framing effect vs. older adults not demonstrating the framing effect). Responses to this question were initially copied verbatim from participant materials and were sorted into the correct table. Once all the responses were recorded, the principal investigator grouped similar responses together and named them by using the most frequently cited phrase within the response. The tables of results from this question are presented in Appendix O.

For the first comparison, between older adults who did and did not demonstrate the framing effect, several patterns emerged. Those older adults who demonstrated the framing effect considered living longest at the 5-year mark as the most important decision making factor, whereas those who did not demonstrate the framing effect considered personal and vicarious experience as the most important decision making factor. These results complement the results of Research Question 3a, which found that older adults who demonstrated the framing effect most often discussed comparative decision making factors, whereas those older adults who did not demonstrate the framing effect most often discussed personal decision making factors. As a group, older adults that did not demonstrate the framing effect had significantly more factors that were important in their decision making process, as compared to older adults who demonstrated the framing effect. For example, none of the older adults who demonstrated the framing effect talked about cost, severity of the cancer, time spent at home during the treatment, taking care of a spouse during treatment, or selecting the treatment that is less damaging to the body as important in their decision.

The second comparison examined responses between younger adults who demonstrated the framing effect and those that did not. Choosing the treatment with the longest life expectancy at the 5-year mark was most important to those younger adults who demonstrated the 
framing effect, whereas those who did not demonstrate the framing effect considered the overall survival rate at each data point as most important in their decision. This result complements the results of Research Question 3b, which found that both groups of younger adults (those that did and did not demonstrate the framing effect) were likely to discuss comparative decision making factors, a result which provided little insight into factors that differentiate younger adults that demonstrated the framing effect from those who did not. The results of this exploratory analysis indicate that, although both groups of younger adults were using comparative decision making factors, those who did not demonstrate the framing effect were basing their decision on survival rates at each data point, thus potentially engaging in a more thorough analysis of the two treatment options.

The final comparison for this exploratory research question examined differences in most important decision making factors between younger and older adults. The most notable difference between younger and older adult responses to this question was that older adults were more likely to cite personal and vicarious factors as most important in their decision, whereas younger adults were more likely to cite life expectancy at the 5-year mark as most important. Additionally, older adults were noticeably less concerned than younger adults about dying through the treatment (e.g., on the operating table).

\section{Discussion}

Several results were obtained for younger and older adult participants that clarified how older and younger adults considered the presented information, what pieces of information were important in their decision, and whether they were susceptible to the framing effect. These results are reviewed separately for each age group, followed by a discussion of age-related differences in the results. 


\section{Older Adult Decisional Process \& Outcome}

Although the findings indicated that the majority of older adults did not demonstrate a framing effect in both the cumulative or interval probability formats, the results of the qualitative analyses allow us to comment on the process by which individual older adults arrived at their decisions, and how this differed between framers and non-framers. For the interval probability format, older adults who did not demonstrate the framing effect were more likely to consider personal decision making factors, thus often not considering the presented data. Additionally, those participants who did not demonstrate the framing effect knew, on average, more individuals who had been diagnosed with cancer, as compared to those who demonstrated the framing effect. It is possible that these results were obtained because older adults who had more personal or vicarious experience with the decision did not attempt to fully consider the presented materials, and were thus not effectively exposed to the framing manipulation. However, this finding is still alarming because it may simulate what happens in medical decision making situations where older adults are asked to make decisions for their own health care, rather than under hypothetical conditions. Thus, older adults may not be making their decisions based upon the information presented by their physicians.

This result has several implications regarding older adults' decision making process. First, this finding provides increased support for the role of personal and vicarious experience in older adults' decision making process (c.f., Pierce, 1993). That is, these results suggest that if an older adult has knowledge about a medical decision, gained through either direct or indirect experience, he or she is likely to use that information to guide the final choice, as opposed to processing the presented information before arriving at a decision. If an older adult does not have much personal or vicarious experience to draw on, he or she will likely make an attempt to 
process the presented information. Some researchers have proposed that attempting to understand the presented data may result in biased decisions among older adults due to the notion that older adults have greater difficulty understanding information concerning available options (e.g., Finucane, et al., 2002; Finucane, Mertz, Slovic, \& Schmidt, 2005). That is, if an older adult encounters difficulty with understanding the presented information, he or she may instead rely on a more expedient approach to decision making that is not as taxing on cognitive resources (e.g., use of heuristics).

The present findings indicate that older adults who failed to demonstrate a framing effect were exhibiting the use of an availability heuristic (e.g., Peters, Finucane, MacGregor, \& Slovic, 2000), whereby if there was personal or vicarious information on which to rely, decisions were generally guided by that information. Therefore, one potential process that may be occurring with older adults is that they rely on available information when they have pertinent experience to guide their decision. If this is not the case, they attempt to understand the presented data, have difficulty interpreting the probabilities (e.g., Peters, Vastfjall, Slovic, Mertz, Mazzocco, \& Dickert, 2006) and thus are more susceptible to decisional biases. This finding is opposite to previous researchers' (e.g., Yates \& Patalano, 1999) suggestion that engaging in heuristic-based decision making should be more characteristic of those who demonstrate decisional biases, and not characteristic of those who successfully avoid decisional biases.

The results of the think-aloud data provide information on what pieces of information older adult participants considered in the process of making their decisions. When older adults responded to the question regarding most important factors in their decision, we were able to discern which pieces of information older adults valued most in their decision making. Collapsing across data formats, older adults who demonstrated the framing effect were likely to 
list the length of life at the 5-year mark as the most important factor, whereas those not demonstrating the framing effect were likely to list personal and vicarious experience as the most important factor. This result complements the findings regarding differences in amount of personal and vicarious experience among those who did and did not demonstrate the framing effect. It is interesting to note that those older adults that demonstrated the framing effect relied on a relatively simple factor (length of life at the end of treatment) to guide their decisions. This finding is congruent with older adult decision making research which suggests that older adults may use less cognitively demanding strategies in their decision making process (e.g., Mata, Schooler, \& Rieskamp, 2007).

These results also speak to the role of affect in older adults' decision making process. Both of the "most important" factors employed by older adult framers and non-framers (length of life at the end of treatment and personal/vicarious experience, respectively) could arguably be described as affect laden. That is, one is either making a decision based on the positive or negative treatment experienced by oneself or a friend, or is making the decision based on the "shock" of the number of patients that do not make it to the 5-year mark. There is some research to suggest that, as one ages, judgment and decision making may be more influenced by recall of vivid personal and vicarious experiences, and that these experiences may play a significant role in the construction of older adults' choices (e.g., Blanchard-Fields, Brannan, \& Camp, 1987; Peters et al., 2000).

\section{Younger Adult Decisional Process \& Outcome}

Similar to the findings for older adults, the majority of younger adults did not demonstrate a framing effect in the interval or cumulative probability formats. The lack of a significant framing effect in these formats among both younger and older adults is interesting, 
particularly in light of how robust the framing effect is in the literature (e.g., Levin, Schneider \& Gaeth, 1998). It is possible that the think-aloud procedure influenced whether participants demonstrated a framing effect. Although previous research has found no differences between think-aloud procedures and other decision making procedures (e.g., information search techniques; Johnson, 1993; Hoc \& LePlat, 1983) in terms of steps taken to reach the decision or the final outcome of the decision, it is possible that the think-aloud technique interacted with the framing manipulations. That is, by asking participants to verbalize their thought processes, it is possible that they became aware of any inconsistencies in their choices, and thus were less likely to demonstrate a framing effect. A similar procedure of asking participants to justify their treatment choice has been used to successfully debias the framing effect (Almashat et al., 2008; Miller \& Fagley, 1991), thus making it possible that the think-aloud procedure used in the present study resulted in a similar debiasing effect.

The differences in younger adult framers and non-framers in terms of decisional process and content were less distinct than among older adults. Younger adults in both formats were likely to consider comparative decision making factors, along with one-sided factors, or were likely to base their decision on a single factor. However, younger adults who demonstrated the framing effect were likely to have slightly more education and slightly more knowledge about cancer than those younger adults who did not demonstrate the framing effect. It is unclear how education would influence treatment decision. Education may be indirectly tied to knowledge of cancer. It is possible that for younger adults, not having any knowledge about cancer increased the likelihood that participants took the time to process the presented information and arrive at an unbiased choice. Having increased knowledge may make it more likely that one will make a 
quick decision due to the perception that one already knows the positive and negative effects of each treatment.

The foregoing conclusion is supported by the results we obtained by asking younger adults to list the most important factor in their decision making process. Younger adults demonstrating the framing effect were likely to list the most important factor as choosing whichever treatment had the longest life expectancy at the 5-year mark, whereas those that did not demonstrate the framing effect were likely to list the overall survival rate at each presented data point (post-treatment, one-year, and five-year) as the most important factor.

In combination with the finding that both younger adult framers and non framers considered comparative aspects of the presented data, these results suggest that those that did not demonstrate the framing effect arguably engaged in a more sophisticated analysis of the data by considering survival rates at each data point. This finding is supported by the studies that have successfully eliminated the framing effect among younger adults by asking them to justify their response or consider the positives and negatives of each treatment choice (e.g., Almashat et al., 2008; Kim et al., 2005; Miller \& Fagley, 1991; Woodhead, 2006). This approach might work by potentially "forcing" those who have knowledge about the treatment options to process each option instead of using their knowledge to make a quick decision that does not consider the pros and cons of each option.

Age Differences in Decisional Process \& Outcome

The findings from the qualitative analyses revealed that older and younger adults used different processes to arrive at their decisions and valued pieces of information differently, in terms of what was most important in their decision. These differences could be related to age differences in information processing, though it is also possible that the obtained differences are 
due to the notable difference in level of education achieved by our older and younger adult sample. Overall, older adults were more likely to consider personal decision making factors and one-sided factors, or base their decision on a single factor. This is contrasted with younger adults, who were more likely to compare the data for each of the presented treatment options. This finding complements the notion that older adults may rely more heavily on available information during decision making. Even though older and younger adults in the present study knew approximately the same number of individuals who had been diagnosed with cancer, older adults may have relied on their personal and vicarious experience more heavily because it was potentially more easy to access, and not because they necessarily had more personal or vicarious experiences to draw upon, as compared to younger adults. Additionally, older adults may have been more swayed by personal and vicarious experience due to the affective component of personal experience.

Taken together, results indicate that for older adults, prior experience is potentially helpful in reducing biased medical decisions because without it, older adults may attempt to process the presented data but not have the necessary fluid intelligence skills to adequately consider the information. This may lead older adults to rely on one piece of presented information that they can easily understand, instead of considering the entire array of presented data. For younger adults, relying on prior knowledge may actually increase the chance of making a biased decision, because they may be more likely to skip over important information due to their perception that they know the pros and cons of each choice.

The exploratory research questions helped to confirm some of the findings of existing research on differences among younger and older adults on use of the think-aloud procedure. We found that there were no significant differences in time to decision between younger and 
older adults, thus contradicting results indicating that older adults may make treatment decisions more quickly than younger adults (Leventhal et al., 1993; Meyer et al., 1995). We also found that all participants made few errors in interpreting the data, and older adults were more likely to make errors in only one of the four scenarios (survival frame, interval probability format). This result is in contrast to other studies, which have found that older adults consistently make more comprehension errors than younger adults (e.g., Finucane et al., 2002). However, many of our older adult participants did not reference any of the presented data, thus they were not required to demonstrate comprehension of the data, as has been the case in most other studies that have examined comprehension among older adults. Additionally, we found that younger adults were more likely to reference the presented data than older adults, and that those demonstrating the framing effect were more likely to reference the data than those not demonstrating the framing effect.

As an indication of how "well" each age group performed with the think-aloud procedure, number of words was recorded for each transcript. One might expect that fewer words would indicate that a participant did not adequately comply with the think-aloud procedure, as participants were instructed to verbalize all thought processes, whether or not they thought they were relevant to the presented decision. It is also possible that fewer words on a think-aloud protocol would indicate that a participant was not proficient at verbalizing all of his or her thoughts, and was only providing a sample of thoughts during the decision scenario. We found that older adults used significantly fewer words for only one scenario (mortality frame, interval probability format), thus supporting results from Johnson (1993) who also found no word count differences between younger and older adults on their think-aloud task. 


\section{Limitations}

The presented results must be considered in light of some limitations. First, the typographical errors in the interval probability format may invalidate the results obtained with this format for both the tests of statistical significance (chi-square, logistic regression) and the qualitative analyses. When considering the impact of these errors, it is important to note that the qualitative data suggest that participants in both age groups were not considering the data at a level of detail that would reveal the errors. That is, none of the participants' transcripts indicated that comparisons of the presented data were being made within the scenario, though several participants compared the data for surgery and radiation at each data point. Additionally, no participants made any comments about the errors while performing the think-aloud task.

A second limitation is the possibility that the think-aloud procedure compromised the framing effect manipulation. Since no other studies have used a think-aloud procedure to examine the framing effect, it is unknown whether use of a think-aloud procedure reduces the proportion of individuals who demonstrate the framing effect. Given that one debiasing procedure that has been successful is to ask participants to consider the positives and negatives of each treatment option (e.g., Miller \& Fagley, 1991), it is possible that asking participants to explain their decision while they are making it would also decrease the probability of a framing effect. It could also be argued that the use of a within-subjects design was a limitation, in that it could have led to a similar reduction in the proportion of individuals demonstrating the framing effect. Although the survival and mortality worded scenarios of each format were never juxtaposed, it is possible that the presentation of alternating survival and mortality frames may have been transparent to participants, thus leading to our finding of consistency in choice across frames. 
A third limitation is that participants were asked to pretend they were faced with the medical decision in the scenarios, which may limit the external validity of these findings. There is some research to suggest that patients who are making a decision for a current medical condition are not as susceptible to framing effect manipulations as those who are asked to imagine that they have the condition (Siminoff \& Fetting, 1989), possibly because those who are actually making the decision are more thorough in their consideration of the information than those who are asked to pretend they had a certain medical condition. This might lead to a decisional process that is more comprehensive than what was reflected in our results.

Additionally, cohort differences cannot be ruled out as a potential reason for age-related differences that were found in the current study. Given that the design of the study was crosssectional, it is possible that future generations of older adults will rely more heavily on an analytic decision making mode to make medical decisions, and will consider personal experience less important than the current generation of older adults. Similarly, it is possible that the agerelated differences noted in the qualitative analyses were due primarily to the educational differences between our younger and older adult sample. Future generations of older adults will likely have higher educational attainment. Thus, the types of age-related differences that we observed may be attenuated when educational attainment is relatively equal between younger and older adult samples.

\section{Conclusions}

Several conclusions can be drawn regarding age-related differences in decisional processes when presented with hypothetical medical decisions. First, we found that older adults appear to be more influenced than younger adults by personal or vicarious experience that is 
relevant to the decision, and are less likely to reference the data presented in the scenario. In contrast, younger adults appear to take a more analytic approach to decision making.

Given relatively equal amounts of vicarious experience with the content of the decision, older adults are more likely to use heuristics than younger adults and are more likely to not consider all of the presented data. When older adults attempted to consider all of the presented data, they were more likely to demonstrate biased decision making and were more likely to base their decision on simpler pieces of information. This is inconsistent with the findings with younger adults who, when faced with a medical decision, are likely to make a biased decision if they are somewhat informed about the subject of the decision. Thus, younger adults who go into a decision "blind" in terms of knowledge of the condition are more likely to engage in a thorough decision making style that reduces the chance of biased decision making.

Taken together, these results suggest that in order to eliminate the framing effect, different strategies would have to be employed for younger than for older adults. Older adults might be asked to consider their personal experience in light of the presented data, whereas younger adults might be asked questions that encourage a thorough evaluation of the presented data (c.f., Almashat et al., 2008; Woodhead, 2006), thus overriding any inclination they may have to skip through the data due to previous knowledge on the topic or previous experience with evaluating statistical outcomes.

\section{Future Directions}

There are no published studies that have used a think-aloud procedure to study the framing effect. The results of the present study suggest that this procedure is quite promising for gaining a better understanding of the decision making process in the context of differentially framed information. Future research might extend the use of a think-aloud procedure to 
investigate other types of decisional biases that may impact medical decisions (e.g, Backlund et al., 2003). It is possible that use of a think-aloud procedure would illuminate the process that leads different age groups to demonstrate decisional biases, thus leading to more information regarding how older and younger adults might successfully avoid medical decision making biases.

There are interesting directions for further research on older adults' susceptibility to decisional biases. For instance, our results suggest that having more personal or vicarious experience may make it less likely that one will demonstrate a decisional bias. Since this result is tentative, it could be tested more rigorously both within the medical decision domain and in other arenas where older adults may be affected by their poor decision making. Although the strategy of relying on personal or vicarious experience may increase the likelihood of decision making uninformed by the presented data, it is possible that uncovering the ways that older adults are approaching medical decisions will lead to better informed intervention attempts. Researchers might also explore the potential debiasing effect of such a procedure. Our results also suggest new methods that might be used to eliminate the framing effect, particularly among older adults. Although researchers have had success with eliminating the framing effect among younger adults by asking them to consider the pros and cons of each treatment choice (e.g., Almashat et al., 2008; Kim et al., 2005; Miller \& Fagley, 1991) this procedure has worked less well with older adults (e.g., Woodhead, 2006). There may be no benefit to asking older adults to consider the data on their own, as it is possible that they will not comprehend the presented information, or may not make the effort to do so. Additionally, it appears likely that older adults will rely on previous personal and vicarious experience if they have it. Thus, 
piloting a debiasing procedure for older adults that helps older adults consider the presented data in light of their personal experience is a possible next step. 


\section{References}

Allaire, J. C., \& Marsiske, M. (1999). Everyday cognition: Age and intellectual ability correlates. Psychology and Aging, 14, 627-644.

Almashat, S., Ayotte, B., Edelstein, B., \& Margrett, J. (2008). Framing effect debiasing in medical decision making. Patient Education and Counseling, 71, 102-107.

American Cancer Society. (2005). The patient self-determination act (PSDA). Retrieved March 20, 2007, from http://www.cancer.org/docroot/MIT/content/MIT_3_2X_The_ Patient_Self-Determination_Act.asp

Backlund, L., Skåner, Y., Montgomery, H., Bring, J., \& Strender, L. E. (2003). Doctors’ decision processes in a drug-prescription task: The validity of rating scales and think-aloud reports. Organizational Behavior and Human Decision Processes, 91, 108-117.

Berg, C. A., Johnson, M., Meegan, S. P., \& Strough, J. (2003). Collaborative problem solving interactions in young and old married couples. Discourse Processes, 35, 33-58.

Blanchard-Fields, F., Brannan, J. R., \& Camp, C. J. (1987). Alternative conceptions of wisdom: An onion-peeling exercise. Educational Gerontology, 13, 497-503.

Blanchard-Fields, F., Chen, Y., \& Norris, L. (1997). Everyday problem solving across the adult life span: Influence of domain specificity and cognitive appraisal. Psychology and Aging, 12, 684-693.

Blanchard-Fields, F., Mienaltowski, A., \& Seay, R. B. (2007). Age differences in everyday problem-solving effectiveness: Older adults select more effective strategies for interpersonal problems. Journals of Gerontology Series B: Psychological Sciences and Social Sciences, 62, P61-64. 
Bradley, E. H., Wetle, T., Horwitz, S. M. (1998). The patient self-determination act and advance direction completion in nursing homes. Archives of Family Medicine, 7, 417-423.

Brinkman, J. A. (1993). Verbal protocol accuracy in fault diagnosis. Ergonomics, 36, 1381-1397. Centers for Disease Control and Prevention. (2002). The national nursing home survey: 1999 summary. Retrieved March 30, 2007, from http://www.cdc.gov/nchs/fastats/nursingh.htm

Chen, Y., \& Sun, Y. (2003). Age differences in financial decision-making: Using simple heuristics. Educational Gerontology, 29, 627-635.

Cornelius, S. W., \& Caspi, A. (1987). Everyday problem solving in adulthood and old age. Psychology and Aging, 2, 144-153.

Diehl, M., Willis, S. L., \& Schaie, K. W. (1995). Everyday problem solving in older adults: Observational assessment and cognitive correlates. Psychology and Aging, 10, 478-491.

D’Zurilla, T. J., \& Nezu, A. (1982). Social problem solving in adults. In P. C. Kendall (Ed.), Advances in cognitive-behavioral research and therapy (Vol. 1, pp. 201-274). New York: Academic Press.

D’Zurilla, T. J. (1986). Problem-solving therapy: A social approach to clinical intervention. New York: Springer.

Ericsson, K. A., \& Simon, H. A. (1984). Protocol analysis: Verbal reports as data. Cambridge, MA: The MIT Press.

Ericsson, K. A., \& Simon, H. A. (1993). Protocol analysis: Verbal reports as data. Cambridge, MA: The MIT Press. 
Finucane, M. L., Slovic, P., Hibbard, J. H., Peters, E., Mertz, C. K., \& MacGregor, D. G. (2002). Aging and decision-making competence: An analysis of comprehension and consistency skills in older versus younger adults considering health-plan options. Journal of Behavioral Decision Making, 16, 141-164.

Finucane, M. L., Mertz, C. K., Slovic, P., \& Schmidt, E. S. (2005). Task complexity and older adults' decision-making competence. Psychology and Aging, 20, 71-84.

Hastie, R., \& Dawes, R. M. (2001). Rational choice in an uncertain world. London: Sage Publications.

Hoc, J. M., \& Leplat, J. (1983). Evaluation of different modalities of verbalization in a sorting task. International Journal of Man-Machine Studies, 18, 283-306.

Hughes, K. K. (1993). Decision making by patients with breast cancer: The role of information in treatment selection. Oncology Nursing Forum, 4, 623-628.

Hux, J. E., \& Naylor, C. D. (1995). Communicating the benefits of chronic preventive therapy: Does the format of efficacy data determine patients' acceptance of treatment? Medical Decision Making, 15, 152-157.

Johnson, M. M. S. (1993). Thinking about strategies during, before, and after making a decision. Psychology and Aging, 8, 231-241.

Kim, S., Goldstein, D., Hasher, L., \& Zacks, R. T. (2005). Framing effects in younger and older adults. Journal of Gerontology: Psychological Sciences, 60B, P215-P218.

Kim, S., \& Hasher, L. (2005). The attraction effect in decision making: Superior performance by older adults. The Quarterly Journal of Experimental Psychology, 58A, 120-133. 
King, A. C., Rejeski, W. J., \& Buchner, D. M. (1998). Physical activity interventions targeting older adults: A critical review and recommendations. American Journal of Preventive Medicine, 15, 315-333.

Kühberger, A. (1998). The influence of framing on risky decisions: A meta-analysis. Organizational Behavior and Human Decision Processes, 75, 23-55.

Leventhal, H., Leventhal, E., Schaefer, P., \& Easterling, D. (1993). Conservation of energy, uncertainty reduction and swift utilization of medical care among the elderly. Journal of Gerontology, 28, 78-86.

Levin, I. P., Schneider, S. L., \& Gaeth, G. J. (1998). All frames are not created equal: A typology and critical analysis of framing effects. Organizational Behavior and Human Decision Processes, 76, 149-188.

Marsiske, M., \& Margrett, J. A. (2006). Everyday problem solving and decision making. In J. Birren and K. Schaie (Eds.), Handbook of the Psychology of Aging (pp.315-342). New York: Academic Press.

Marsiske, M., \& Willis, S. L. (1995). Dimensionality of everyday problem solving in older adults. Psychology and Aging, 10, 269-283.

Marteau, T. M. (1989). Framing of information: Its influence upon decisions of doctors and patients. British Journal of Social Psychology, 28, 89-94.

Mata, R., Schooler, L. J., \& Rieskamp, J. (2007). The aging decision maker: Cognitive aging and the adaptive selection of decision strategies. Psychology and Aging, 22, 796-810.

McKee, D. R. (2001). The effects of framing on younger and older adults' medical decision making. Unpublished doctoral dissertation, West Virginia University. 
McNeil, B. J., Pauker, S. G., Sox, H. C., \& Tversky, A. (1982). On the elicitation of preferences for alternative therapies. The New England Journal of Medicine, $306,1259-1262$.

McNeil, B. J., Pauker, S. G., \& Tversky, A. (1988). On the framing of medical decisions. In D. E. Bell, H. Raiffa, and A. Tversky (Eds.), Decision-making: Descriptive, Normative, and Prescriptive Interactions. (pp. 562-568). NY: Cambridge.

Medin, D. L., \& Edelson, S. M. (1988). Problem structure and the use of base-rate information from experience. Journal of Experimental Psychology, 117, 68-85.

Meyer, B. J. F., Russo, C., \& Talbot, A. (1995). Discourse comprehension and problem solving: Decisions about the treatment of breast cancer by women across the life span. Psychology and Aging, 10, 84-103.

Miller, P.M., \& Fagley, N.S. (1991). The effects of framing, problem variations, and providing rationale on choice. Personality and Social Psychology Bulletin, 17, $517-522$.

Misselbrook, D., \& Armstrong, D. (2001). Patients' responses to risk information about the benefits of treatment hypertension. British Journal of General Practice, 51, 276-279.

Moxey, A., O'Connell, D, McGettigan, T., \& Henry, D. (2003). Describing treatment effects to patients: How they are expressed makes a difference. Journal of General Internal Medicine, 18, 948-959.

Offredy, M., \& Meerabeau, E. (2005). The use of 'think-aloud' technique, information processing theory and schema theory to explain decision making processes of general practitioners and nurse practitioners using patient scenarios. Primary Health Care Research and Development, 6, 46-59. 
Park, D. C. (1999). Aging and the controlled and automatic processing of medical information and medical intentions. In D.C. Park, R.W. Morrell, \& K. Shifren (Eds.), Processing of Medical Information in Aging Patients (pp. 3-22).

London: Lawrence Erlbaum Associates, Inc.

Peters, E., Finucane, M. L., MacGregor, D. G., \& Slovic, P. (2000). The bearable lightness of aging: Judgment and decision processes in older adults. In P. C. Stern \& L. L. Carstensen (Eds.), The Aging Mind: Opportunities in Cognitive Research (pp. 144-165).

Washington, DC: National Academy Press.

Peters, E., Vastfjall, D., Slovic, P., Mertz, C. K., Mazzocco, K., \& Dickert, S. (2006). Numeracy and decision making. Psychological Science, 17, 407-413.

Pierce, P. F. (1993). Deciding on breast cancer treatment: A description of decision behavior. Nursing Research, 42, 22-28.

Piñon, A., \& Gambara, H. (2005). A meta-analytic review of framing effect: Risky, attribute, and goal framing. Psicothema, 17, 325-331.

Riggle, E. D. B., \& Johnson, M. M. S. (1996). Age difference in political decision making: Strategies for evaluating political candidates. Political Behavior, 18, 99-118.

Salthouse, T. A., \& Babcock, R. L. (1991). Decomposing adult age differences in working memory. Developmental Psychology, 27, 763-776.

Salthouse, T. A. (1992). Why do adult age differences increase with task complexity? Developmental Psychology, 28, 905-918.

Salthouse, T. A. (1996). The processing-speed theory of adult age differences in cognition. Psychological Review, 103, 403-428. 
Schaie, K. W. S. (1994). The course of adult intellectual development. American Psychologist, 49, 304-313.

Schaie, K. W. S., \& Willis, S. L. (1996). Adult Development and Aging (4 ${ }^{\text {th }}$ ed.). New York: HarperCollins.

Schuller, K. L. (2006). The framing effect and breast cancer treatment options: Do individual characteristics play a role? Unpublished doctoral dissertation, West Virginia University.

Siminoff, L. A., \& Fetting, J. H. (1989). Effects of outcome framing on treatment decisions in the real world: Impact of framing on adjuvant breast cancer decisions. Medical Decision Making, 9, 262-271.

Simon, H. A. (1957). Models of man - social and rational. New York: John Wiley and sons.

Smith, S. M., \& Levin, I. P. (1996). Need for cognition and choice framing effects. Journal of Behavioral Decision Making, 9, 283-290.

Spaniol, J., \& Bayen, U. J. (2005). Aging and conditional probability judgments: A global matching approach. Psychology and Aging, 20, 165-181.

Tariq, S. H., Tumosa, N., Chibnall, J. T., Perry, M. H., \& Morley, J. E. (2006). Comparison of the St. Louis University Mental Status Examination and the Mini-Mental State Examination for detecting dementia and mild neurocognitive disorder - A pilot study. American Journal of Geriatric Psychiatry, 14, 900-910.

Tengs, T. O. (1987). The framing effect in medical decision making. Unpublished master's thesis, University of Massachusetts.

Thornton, W. J., \& Dumke, H. A. (2005). Age differences in everyday problem-solving and decision-making effectiveness: A meta-analytic review. Psychology and Aging, 20, 8599. 
Tversky, A., \& Kahneman, D. (1973). Availability: A heuristic for judging frequency and probability. Cognitive Psychology, 5, 207-232.

Tversky, A., \& Kahneman, D. (1974). Judgment under uncertainty: Heuristics and biases. Science, 185, 1124-1131

Tversky, A., \& Kahneman, D. (1981). The framing of decisions and the psychology of choice. Science, 185, 1124-1131.

U.S. Administration on Aging. (2003). A profile of older Americans: 2003. Retrieved February 2, 2007, from http://www.aoa.gov/prof/statistics/profile/2003/2_pf.asp.

Walker, N., Fain, W. B., Fisk, A. D., \& McGuire, C. L. (1997). Aging and decision making: Driving-related problem-solving. Human Factors, 39, 438-444.

Watson, J. B. (1920). Is thinking nearly the action of language mechanisms? British Journal of Psychology, 11, 87-104.

Willis, S. L., \& Schaie, K. W. (1986). Everyday cognition: Taxonomic and methodological considerations. In J. M. Puckett \& H. W. Reese (Eds.), Mechanisms of everyday cognition (pp. 33-54). Hillsdale, NJ: Lawrence Erlbaum.

Woodhead, E. L., \& Edelstein, B. A. (2006, November). Debiasing the framing effect in younger and older adults' medical decision-making. Poster presented at the annual meeting of the Gerontological Society of America, Dallas TX.

Yates, J. F., \& Patalano, A. L. (1999). Decision making and aging. In D.C. Park, R.W. Morrell, \& K. Shifren (Eds.), Processing of Medical Information in Aging Patients (pp. 3-22). London: Lawrence Erlbaum Associates, Inc.

Zwahr, M. D. (1994). A model to predict decisions about postmenopausal estrogen replacement therapy. Unpublished doctoral dissertation, University of Georgia, Athens. 
Zwahr, M. D., Park, D. C., \& Shifren, K. (1999). Judgments about estrogen replacement therapy: The role of age, cognitive abilities and beliefs. Psychology and Aging, 14, 179-191. 


\section{Appendix A}

\section{Think-Aloud Instructions}

"In this experiment we are interested in what you think about when you make a choice between two hypothetical medical treatments. In order to do this, I am going to ask you to THINK ALOUD as you make your decision. What I mean by think aloud is that I want you to tell me EVERYTHING you are thinking from the time you finish reading the scenario until you make a choice. I would like you to talk aloud CONSTANTLY from the time you finish reading the scenario until you have made your final choice. I don't want you to try to plan out what you say or try to explain to me what you are saying. Just act as if you are alone in the room speaking to yourself. It is most important that you keep talking. If you are silent for a long period of time I will ask you to talk. Do you have any questions about what I'm asking you to do? We will begin with some practice problems." 


\section{Appendix B}

\section{Think-Aloud Practice Problems}

"First, I'm going to demonstrate a think-aloud practice problem. If I were asked to multiply $21 \mathrm{x}$ 6 and think aloud while doing it, it might sound like this (model the think-aloud approach).

Now we'll do two more problems so you can practice thinking aloud. I'll be timing you to make sure you talk continuously without any long breaks. We might repeat the problems if more practice is needed.

How many windows are/were there in your parent's house?

Name 20 animals. I will keep count for you" 


\section{Appendix C \\ Participant Instructions}

The following pages contain specific information about cancer treatments at several Chicago area hospitals. Each hospital has its own doctors and policies regarding patient care, approaches to treatment, and different survival rates for the various types of treatment. For each hospital, please indicate whether you prefer surgery or radiation therapy. Below are general descriptions of the treatments.

Surgery for lung cancer involves an operation on the lungs. Most patients are in the hospital for two to three weeks and have some pain around their incisions; they spend a month or so recuperating at home. After that they generally feel fine.

Radiation therapy for lung cancer involves the use of radiation to kill the tumor and requires coming to the hospital about four times a week for six weeks. Each treatment takes a few minutes, and during the treatment patients lie on a table as if they were having an x-ray. During the course of treatment, some patients develop nausea and vomiting, but by the end of six weeks they generally feel fine.

Thus, after the initial six weeks, patients treated with either surgery or radiation therapy feel about the same.

Please read the scenarios in the order they appear. Please think-aloud about all the information that you would hypothetically consider with a decision of this magnitude, including personal experiences and the presented data for the scenario you are reading. 
Appendix D

Stimulus Materials

(Cumulative Probability Information; Survival Wording)

North Hospital

Of 100 patients having radiation therapy, all patients live through the treatment, 77 patients live for more than one year, and 22 patients live for more than five years.

Of 100 patients having surgery, 90 patients live through treatment, 68 patients live for more than one year, and 34 patients live for more than five years.

**Please think-aloud while you make your choice between radiation and surgery. Please think only of the numbers and information from this scenario. You may discuss any information that would help you make this decision, including personal information and the information in the Participant Instructions. 
(Cumulative Probability Information; Mortality Wording)

$\underline{\text { South Hospital }}$

Of 100 patients having surgery, 10 die during the treatment, 32 die by one year, and 66 die by 5 years.

Of 100 patients having radiation therapy, no patients die during treatment, 23 die by one year, and 78 die by 5 years.

**Please think-aloud while you make your choice between radiation and surgery. Please think only of the numbers and information from this scenario. You may discuss any information that would help you make this decision, including personal information and the information in the Participant Instructions. 
(Interval Probability Information; Survival Wording)

\section{East Hospital}

Of 100 patients having surgery, 90 patients live until the end of treatment, 78 patients live through treatment but less than one year, 44 patients live for one to five years, and 10 patients live longer than five years.

Of 100 patients having radiation therapy, all patients live through the end of treatment, 77 live through the treatment but less than one year, 22 patients live for one to five years, and 5 patients live for more than five years.

***Please think-aloud while you make your choice between radiation and surgery. Please think only of the numbers and information from this scenario. You may discuss any information that would help you make this decision, including personal information and the information in the Participant Instructions. 
(Interval Probability Information; Mortality Wording)

West Hospital

Of 100 patients having radiation therapy, no patients die by the end of treatment, 32 die in the time interval between treatment and one year, 55 die in the interval between one and five years, and 22 die sometime after five years.

Of 100 patients having surgery, 10 patients die by the end of treatment, 22 patients die in the interval between treatment and one year, 34 patients die in the interval between one and five years, and 34 patients die sometime after five years.

***Please think-aloud while you make your choice between radiation and surgery. Please think only of the numbers and information in this scenario. You may discuss any information that would help you make this decision, including personal information and the information in the Participant Instructions. 


\title{
Appendix E
}

\section{Treatment Choice Questionnaire}

Which hypothetical cancer treatment would you prefer at (North/South/East/West) Hospital? Please circle one:

Surgery

\author{
Radiation Therapy
}




\section{Appendix F}

\section{Demographic Questionnaire}

\section{Background Information}

Age:

Sex: (circle one) Male Female

Years of Education:

Marital Status: (circle one)

Single $\quad$ Married Separated Divorced Widowed

Ethnicity: (race)

Caucasian (White)

African American (Black)

Asian American

Hispanic

Pacific Islander

Native American (American Indian/Alaskan Native)

What is your current or former occupation (for older adult participants only)?

What is/are the occupations of your parents (for undergraduate participants only)?

Have you ever watched TV programs about individuals with cancer? Yes No

If so, how important were these TV programs in influencing how you currently think about cancer?

not at all important $\quad$ very important

Have you ever listened to radio programs about individuals with cancer? Yes No

If so, how important were these radio programs in influencing how you currently think about cancer?

not at all important $\quad$ very important


Have you ever read articles in magazines about individuals with cancer? Yes No

If so, how important were these articles in influencing how you currently think about cancer?

not at all important $\quad$ very important

\section{Personal Health Information}

Have you ever been diagnosed with a chronic illness (e.g., heart disease, diabetes, arthritis)? Yes No

If Yes, please write-in the names of the illnesses:

How long has it been since you were examined by a doctor?

Please rate your current health status: 1 . Poor

2. Fair

3. Average

4. Good

5. Excellent

Have you ever been diagnosed with cancer? Yes No

If yes, when were you diagnosed?

What type of cancer?

What treatment did you receive?

\section{Vicarious Experience Information}

Please indicate how many individuals you know that have ever been diagnosed with any type of cancer:

What types of cancer?

How many of these individuals were friends? extended family? immediate family?

Have you ever discussed the experience and/or treatment of cancer with someone who had cancer? Yes No 
If so, please rate how involved you were in this person's life during their experience with cancer:

$$
\text { 1---------2------------3------------4-----------5 }
$$

not at all involved very involved

\section{$\underline{\text { Knowledge of Cancer }}$}

1. More than $87 \%$ of lung cancers are smoking related:

True

False

2. Each year, more Americans die from breast, prostate, and colorectal cancers combined than from lung cancer.

True

False

3. The scarring caused by tuberculosis can cause lung cancer to develop.

True

False

4. Most people diagnosed with lung cancer are 35 to 55 years old.

True

False

5. Lung cancer is the leading cause of death in the United States and worldwide.

True

False

6. Men are 1.5 times more likely to develop lung cancer than women.

True

False

7. Lung cancer cases among women are on the rise.

True

False

8. Quitting smoking significantly reduces a person's risk for lung cancer.

True

False

9. Lung cancer kills more African Americans than any other cancer.

True

False 


\section{Appendix G}

\section{Saint Louis University Mental Status (SLUMS) Examination}

1. What day of the week is it? (1 point for the right answer)

2. What is the year? (1 point)

3. What state are we in? (1 point)

4. Please remember these five objects. I will ask you what they are later: apple, pen, tie, house, car. (No points yet)

5. You have $\$ 100$ and you go to the store and buy a dozen apples for $\$ 3$ and a tricycle for $\$ 20$.

o How much did you spend? (1 point)

o How much do you have left? (2 points)

6. Please name as many animals as you can in one minute. (No point for naming $0-5 ; 1$ point for naming 5-10; 2 points for naming 10-15; and 3 points for naming more than 15.)

7. What were the five objects I asked you to remember? (1 point for each object remembered.)

8. I am going to say a series of numbers and I would like you to give them to me backwards. For example, if I say 42, you would say 24.

- 87 (0 points)

o 649 (1 point)

o 8537 (1 points) 
9. (Draw circle.) This circle represents a clock face. Please put in the hour markers and the time at ten minutes to eleven o'clock.

o (2 points for hour markers labeled correctly)

o (2 points for correct time)

10. (Show a triangle, a square and a rectangle.) Please place an $\mathrm{X}$ in the triangle. (1 point)

11. Which of those objects is the largest? (1 point)

12. I am going to tell you a story. Please listen carefully because afterward, I'm going to ask you some questions about it.

Jill was a very successful stockbroker. She made a lot of money in the stock market. She then met Jack, a devastatingly handsome man. She married him and had three children. They lived in Chicago. She then stopped working and stayed at home to bring up her children. When they were teenagers, she went back to work. She and Jack lived happily ever after.

o What was the female's name? (2 points)

o When did she go back to work? (2 points)

o What work did she do? (2 points)

o What state did she live in? (2 points)

SCORING: Total points out of 30 : 


\section{Appendix $\mathrm{H}$}

\section{Overarching Categories for Qualitative Analyses}

Personal decision making factors

1) Discussion of one friend/family member's experience

2) Discussion of multiple friends/family members' experience

3) Reference to own medical history

4) Concern about effect of decision on others

5) Reference to religious values

6) Consideration of participant's age

Comparative decision making factors (weighing aspects of each treatment)

7) Weighing long-term survival against short-term risk for each treatment

8) Comparison of survival rates for both treatments at each data point

9) Comparison of mortality rates for both treatments at each data point

10) Comparison of survival rates for both treatments at long-term data points (more than one year, 1-5 years, $>5$ years)

11) Comparison of mortality rates for both treatments at long-term data points (more than one year, 1-5 years, $>5$ years)

12) Comparison of mortality rates for both treatments at short-term data points (through treatment, up to one year)

13) Comparison of survival rates for both treatments at short-term data points (through treatment, up to one year)

14) Weighed physical side effects of both treatments

15) Weighed the process of both treatments

One-sided decision making factors

16) Reference to short-term survival statistics for one treatment

17) Reference to short-term mortality statistics for one treatment

18) Reference to long-term survival statistics for one treatment

19) Reference to long-term mortality statistics for one treatment

20) Consideration of physical side effects of one treatment

21) Consideration of the process of one treatment

Treatment specific decision making factors

22) Consideration of rate of recovery

23) References outside information about treatments not provided in stimulus materials

24) Weighing benefits of treatment vs. no treatment vs. alternative methods

25) Consideration of physician recommendation

26) Consideration of the quality of the hospital and the characteristics of the staff

Decision Based Primarily on a Single Factor

27) Vicarious experience directly related to decisional outcome

28) Decision based primarily on certain conditions or qualifications

29) Decision based primarily on wanting to live longer

30) Decision based primarily on wanting to live through the treatment 


\section{Appendix I}

\section{List of All Categories with Participant Examples}

Personal decision making factors

1) Discussion of one friend/family member's experience

a) "My husband never had radiation because they said it was a good, clean operation, but it wasn't. He only lived 6 months."

2) Discussion of multiple friends/family members' experience

a) "My sister had breast cancer and my friend next door had breast cancer and I see how the radiation, how it burned them and stuff."

3) Reference to own medical history

a) "Like when I went in and said, "Here I am"... they said they almost lost me on the first knee because they gave me too much medicine."

4) Concern about effect of decision on others

a) "The longer I live, the better my wife will live because she'll get social security, she won't get cut back if I die."

5) Reference to religious values

a) "I'd just as soon let the guy upstairs take care of me. I don't know I just feel that, in my heart, I feel that the good lord's with me and he's watching me."

6) Consideration of participant's age

a) "At my age of 86 it doesn't matter, I don't think I'd live that much longer."

Comparative decision making factors

1) Weighing long-term survival against short-term risk for each treatment

a) 'I'd rather take a chance with the surgery, because once you're out of there you're better. In other words, you're gambling, you may pass away, but you have a chance of being there without the radiation."

2) Comparison of survival rates for both treatments at each data point

a) 'Now here, there's 78 patients for less than a year, and there's 77, that's the same. Oh I see here 44 live for 1 to 5 , and only $22 \ldots$ right?"

3) Comparison of mortality rates for both treatments at each data point

a) "Well, I would take that first treatment (radiation) where 100 patients having the therapy, they didn't die by the end of the treatment. But 32 die in the interval between treatment and one year, then 55 between 1 and 5 years, and 22 after that. That's a better report than the second one. 10 die by the end of the treatment, and 22 between one year, and 34 in the interval between one and five, and 34 after five."

4) Comparison of survival rates for both treatments at long-term data points (more than one year, 1-5 years, $>5$ years)

a) "I would have the surgery on this one because you live longer. See, 34 patients live more than 5 years and only 22 patients lived."

5) Comparison of mortality rates for both treatments at long-term data points (more than one year, 1-5 years, $>5$ years)

a) "I'm looking at 34 patients die sometime after 5 years, and 22 die sometime after 5 years with the radiation." 
6) Comparison of mortality rates for both treatments at short-term data points (through treatment, up to one year)

a) "What I don't like is 10 patients die by end of treatment. Here's no patients die."

7) Comparison of survival rates for both treatments at short-term data points (through treatment, up to one year)

a) "Because if you take radiation, okay, everybody lives through the treatment program. So you're hoping you're going to live through the surgery, too."

8) Weighed physical side effects of both treatments

a) “And I've seen what radiation does to some people. My sister had breast cancer and my friend next door had breast cancer and I see how the radiation, how it burned them and stuff... And I've had surgeries before, and I know they're not fun, it's really hard to recover, but I don't know."

9) Weighed the process of both treatments

a) "I think the surgery, because it' $d$ be a shorter time... you know, you get in there and they cut you open, you get better and you're gone. With radiation, you've got such a long time... I don't think that's a good idea, I'm not into the pain deal."

One-sided decision making factors

1) Reference to short-term survival statistics for one treatment

a) "Of 100 patients having therapy or radiation, all patients live through the treatment, well that's great, you know."

2) Reference to short-term mortality statistics for one treatment

a) "I would go with surgery even though there is that 10 percent chance you're gonna die through the surgery."

3) Reference to long-term mortality statistics for one treatment

a) "Well, radiation kind of, if only 66 die by 5 years, then does that mean the rest of them live longer than that?"

4) Reference to long-term survival statistics for one treatment

a) "34 patients live for more than 5 years... that sounds like a good percentage."

5) Consideration of physical side effects of one treatment

a) "Of course, I don't know, you may be sick. I don't think I'd want to be sick (with the radiation)."

6) Consideration of the process of one treatment

a) "Surgery is invasive and I think it's harder on the body and harder on the system."

Treatment specific decision making factors

1) References outside information about treatments not provided in stimulus materials

a) "If they open you up, sometimes the air gets to you and it spreads."

2) Consideration of rate of recovery

a) "Not really, it's just the suffering you go through and trying to rehabilitate after the surgery."

3) Weighing benefits of treatment vs. no treatment vs. alternative methods

a) "I might be able to fix it or have them take it out and replace it with an artificial (lung)."

4) Consideration of physician recommendation

a) "I would always base it on what the doctor tells me" 
5) Consideration of the quality of the hospital and the characteristics of the staff

a) "The staff at the hospital would be important too. Like the doctors that you meet, and then the people and the nurses and the quality of the hospital."

Decision Based Primarily on a Single Factor

1) Vicarious experience directly related to decisional outcome

a) "I base the decision mainly on my mom and the other patient that's in our family."

2) Decision based primarily on wanting to live longer

a) "I'm looking at the 34 vs. 22. The longer life is most important to me."

3) Decision based primarily on wanting to live through the treatment

a) "I'd rather take the radiation. I wouldn't want to be lost on the operating table."

4) Decision based primarily on certain conditions or qualifications

a) "So there again, I think I'd go with surgery. Unless it's really in a place where they can't do surgery, then I'd have to go with the radiation." 


\title{
Appendix J
}

\author{
Materials Presented for Kappa Calculation
}

Instructions: For each of the thirty statements listed below, please indicate which of the five overarching categories you think each statement best fits into. The five overarching categories are listed and defined on the corresponding page. Please note that for the current study, participants were asked to choose surgery or radiation to treat lung cancer, and were presented with only those two treatment options.

1) Consideration of rate of recovery

2) Decision based primarily on certain conditions or qualifications*

3) Reference to long-term mortality statistics for one treatment

4) Consideration of physician recommendation

5) _ Comparison of survival rates for both treatments at long-term data points (more than one year, 1-5 years, $>5$ years)

6) __ Discussion of multiple friends/family members' experience

7) Weighing long-term survival against short-term risk for each treatment

8) Consideration of the quality of the hospital and the characteristics of the staff

9) Consideration of participant's age

10) __ Comparison of survival rates for both treatments at each data point

11) D_ Discussion of one friend/family member's experience

12) Weighed the process of both treatments

13) Decision based primarily on wanting to live longer

14) Weighing benefits of treatment vs. no treatment vs. alternative methods

15) __ Decision based primarily on wanting to live through the treatment

16) __ References outside information about treatments not provided in stimulus materials

17) Reference to short-term survival statistics for one treatment

18) Weighed physical side effects of both treatments

19) Reference to long-term survival statistics for one treatment

20) __ Comparison of mortality rates for both treatments at long-term data points (more than one year, 1-5 years, $>5$ years)

21) _ Concern about effect of decision on others

22) Consideration of the process of one treatment

23) ___ Comparison of mortality rates for both treatments at short-term data points (through treatment, up to one year)

24) __ Consideration of physical side effects of one treatment

25) __ Vicarious experience directly related to decisional outcome

26) ___ Comparison of mortality rates for both treatments at each data point

27) __ Reference to religious values

28) __ Reference to short-term mortality statistics for one treatment

29) __ Comparison of survival rates for both treatments at short-term data points (through treatment, up to one year)

30) ___ Reference to own medical history

*Note: An example of a condition or qualification is, "I'll only order the salad if I can get the dressing on the side, otherwise I don't want it." 


\section{Overarching Categories}

\section{Personal Medical Decision Making Factors:}

i. Personal experience

ii. Personal characteristics

iii. Experience of family members and friends

iv. There is no clear indication that the decision was based solely on the statement.

\section{Comparative Decision Making Factors:}

a. Must directly compare aspects of both treatment options presented to participants (surgery and radiation).

i. There is no clear indication that the decision was based solely on the statement.

\section{One-sided Decision Making Factors:}

a. Only aspects of a single treatment (surgery OR radiation, but not both)

i. There is no clear indication that the decision was based solely on the statement.

\section{Treatment Specific Decision Making Factors:}

a. Non-numerical information that is specific to receiving surgery or radiation.

b. Participants could possibly cite outside information

c. There is no clear indication that the decision was based solely on the statement.

\section{Decision Based Primarily on a Single Factor:}

a. Decision was made primarily based on information in the statement, regardless of how many other factors may have been considered. 


\section{Appendix K}

Qualitative Results Table for Research Question 3a - Older Adult Framing Differences

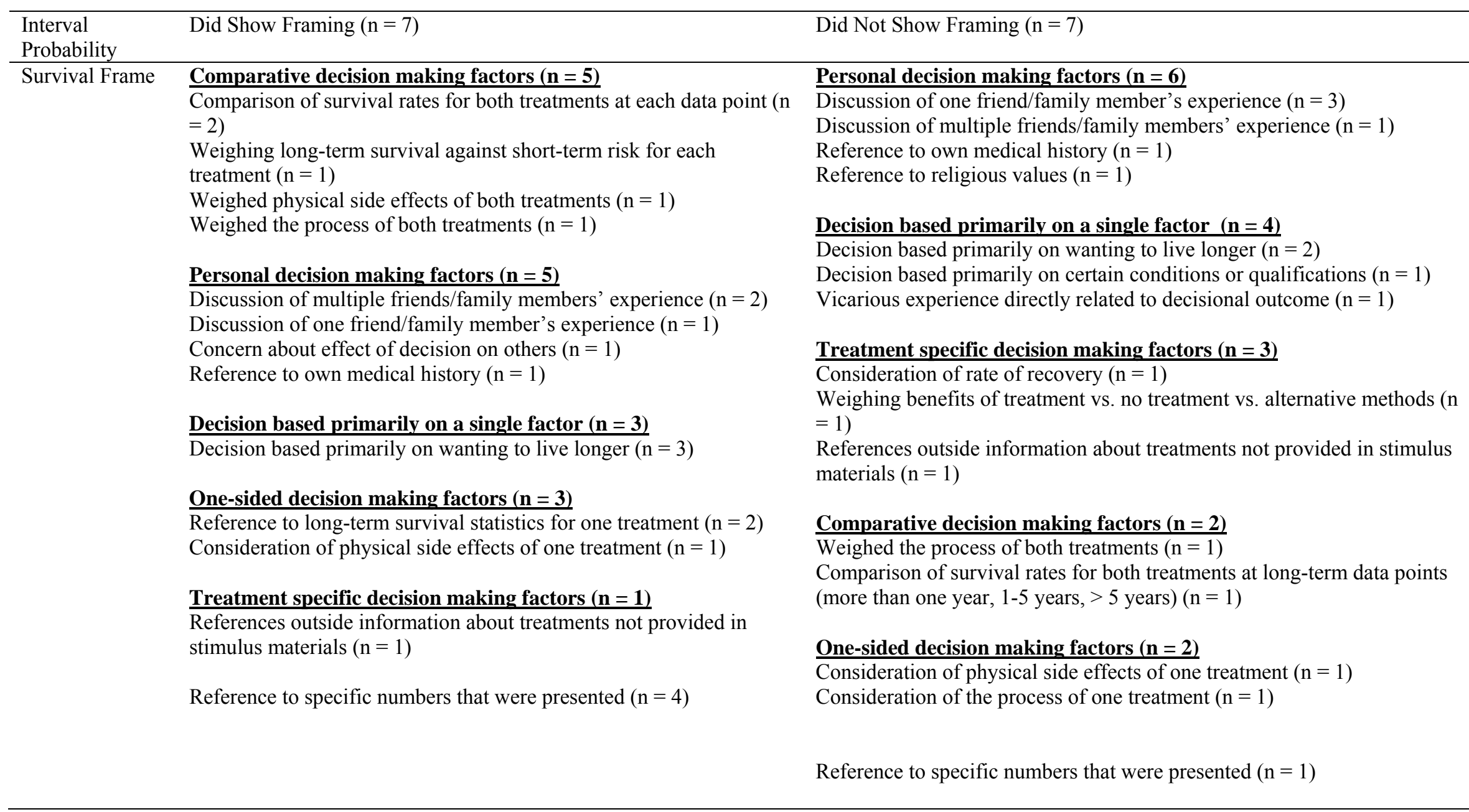




\section{Appendix K (con't)}

Interval

Probability

Mortality Frame

Did Show Framing $(\mathrm{n}=7)$

\section{Comparative decision making factors $(n=5)$}

Comparison of mortality rates for both treatments at each data point $(\mathrm{n}=2)$

Comparison of mortality rates for both treatments at short-term data points (through treatment, up to one year) $(\mathrm{n}=1)$

Comparison of mortality rates for both treatments at long-term data points (more than one year, $1-5$ years, $>5$ years) $(n=1)$

Weighing long-term survival against short-term risk for each treatment $(\mathrm{n}=1)$

\section{One-sided decision making factors $(n=5)$}

Reference to short-term mortality statistics for one treatment $(n=3)$

Reference to long-term mortality statistics for one treatment $(\mathrm{n}=1)$

Consideration of physical side effects of one treatment $(\mathrm{n}=1)$

\section{Decision based primarily on a single factor $(n=4)$}

Decision based primarily on wanting to live through treatment $(\mathrm{n}=2)$

Decision based primarily on wanting to live longer $(n=2)$

\section{Personal decision making factors $(n=1)$}

Discussion of multiple friends/family members' experience $(\mathrm{n}=1)$

Reference to specific numbers that were presented $(n=6)$

\section{Did Not Show Framing $(\mathrm{n}=7)$}

\section{Personal decision making factors $(n=6)$}

Discussion of multiple friends/family members' experience $(\mathrm{n}=2)$

Reference to own medical history $(\mathrm{n}=1)$

Discussion of one friend/family member's experience $(n=1)$

Concern about effect of decision on others $(\mathrm{n}=1)$

Reference to religious values $(\mathrm{n}=1)$

\section{Decision based primarily on a single factor $(n=5)$}

Decision based on primarily wanting to live longer $(n=2)$

Decision based primarily on certain conditions or qualifications $(n=2)$

Vicarious experience directly related to decisional outcome $(n=1)$

\section{Comparative decision making factors $(n=4)$}

Weighing long-term survival against short-term risk for each treatment $(\mathrm{n}=1)$

Comparison of survival rates for both treatments at short-term data points (through treatment, up to one year) $(\mathrm{n}=1)$

Comparison of mortality rates for both treatments at short-term data points (through treatment, up to one year) $(\mathrm{n}=1)$

Weighed physical side effects of both treatments $(n=1)$

\section{One-sided decision making factors $(\mathrm{n}=2)$}

Consideration of physical side effects of one treatment $(n=1)$

Reference to short-term mortality statistics for one treatment $(n=1)$

\section{Treatment specific decision making factors $(\mathrm{n}=1)$}

Consideration of rate of recovery $(n=1)$

Reference to specific numbers that were presented $(n=3)$ 


\section{Appendix K (con't)}

Cumulative

Probability

Survival Frame

Did Not Show Framing $(\mathrm{n}=9)$

Did Show Framing $(\mathrm{n}=9)$

\section{Decision based primarily on a single factor $(n=6)$}

Decision based primarily on certain conditions or qualifications $(n=2)$

Comparison of survival rates for both treatments at long-term data

points (more than one year, $1-5$ years, $>5$ years) $(n=4)$

Comparison of survival rates for both treatments at each data point (n $=1)$

\section{One-sided decision making factors ( $n=4)$}

Reference to short-term survival statistics for one treatment $(n=2)$

Reference to long-term survival statistics for one treatment $(\mathrm{n}=1)$

Consideration of physical side effects of one treatment $(n=1)$

\section{Decision based primarily on a single factor $(n=3)$}

Decision based primarily on wanting to live longer $(\mathrm{n}=2)$

Vicarious experience directly related to decisional outcome $(n=1)$

\section{Personal decision making factors $(n=2)$}

Discussion of one friend/family member's experience $(n=1)$

Discussion of multiple friends/family members' experience $(\mathrm{n}=1)$

Treatment specific decision making factors $(n=1)$

References outside information about treatments not provided in stimulus materials $(\mathrm{n}=1)$

Reference to specific numbers that were presented $(n=7)$

Decision based primarily on wanting to live through the treatment $(\mathrm{n}=$ 2)

Vicarious experience directly related to decisional outcome $(\mathrm{n}=2)$

\section{One-sided decision making factors $(n=6)$}

Consideration of the process of one treatment $(n=2)$

Reference to long-term survival statistics for one treatment $(\mathrm{n}=2)$

Consideration of physical side effects of one treatment $(n=1)$

Reference to short-term survival statistics for one treatment $(n=1)$

\section{Personal decision making factors $(n=4)$}

Discussion of multiple friends/family members' experience $(\mathrm{n}=2)$

Reference to own medical history $(\mathrm{n}=1)$

Concern about effect of decision on others $(n=1)$

\section{Treatment specific decision making factors $(n=4)$}

Weighing benefits of treatment vs. no treatment vs. alternative methods $(\mathrm{n}=1)$

Consideration of rate of recovery $(n=1)$

References outside information about treatments not provided in stimulus materials $(\mathrm{n}=1)$

Consideration of physician recommendation $(n=1)$

\section{Comparative decision making factors $(n=2)$}

Weighing long-term survival against short-term risk for each treatment $(\mathrm{n}=2)$

Reference to specific numbers that were presented $(n=3)$ 


\section{Appendix K (con't)}

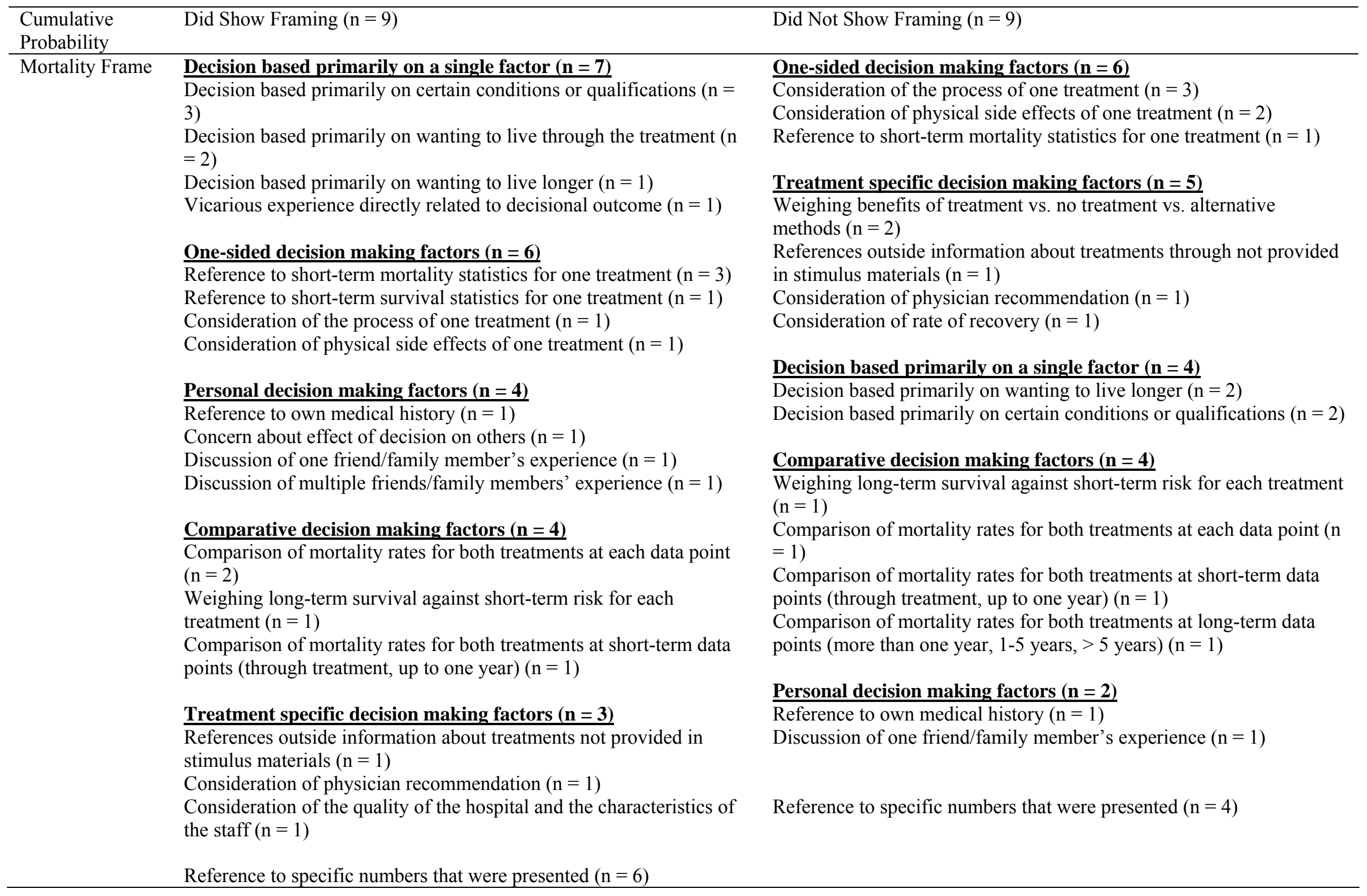




\section{Appendix L}

Qualitative Results Table for Research Question 3b - Younger Adult Framing Differences

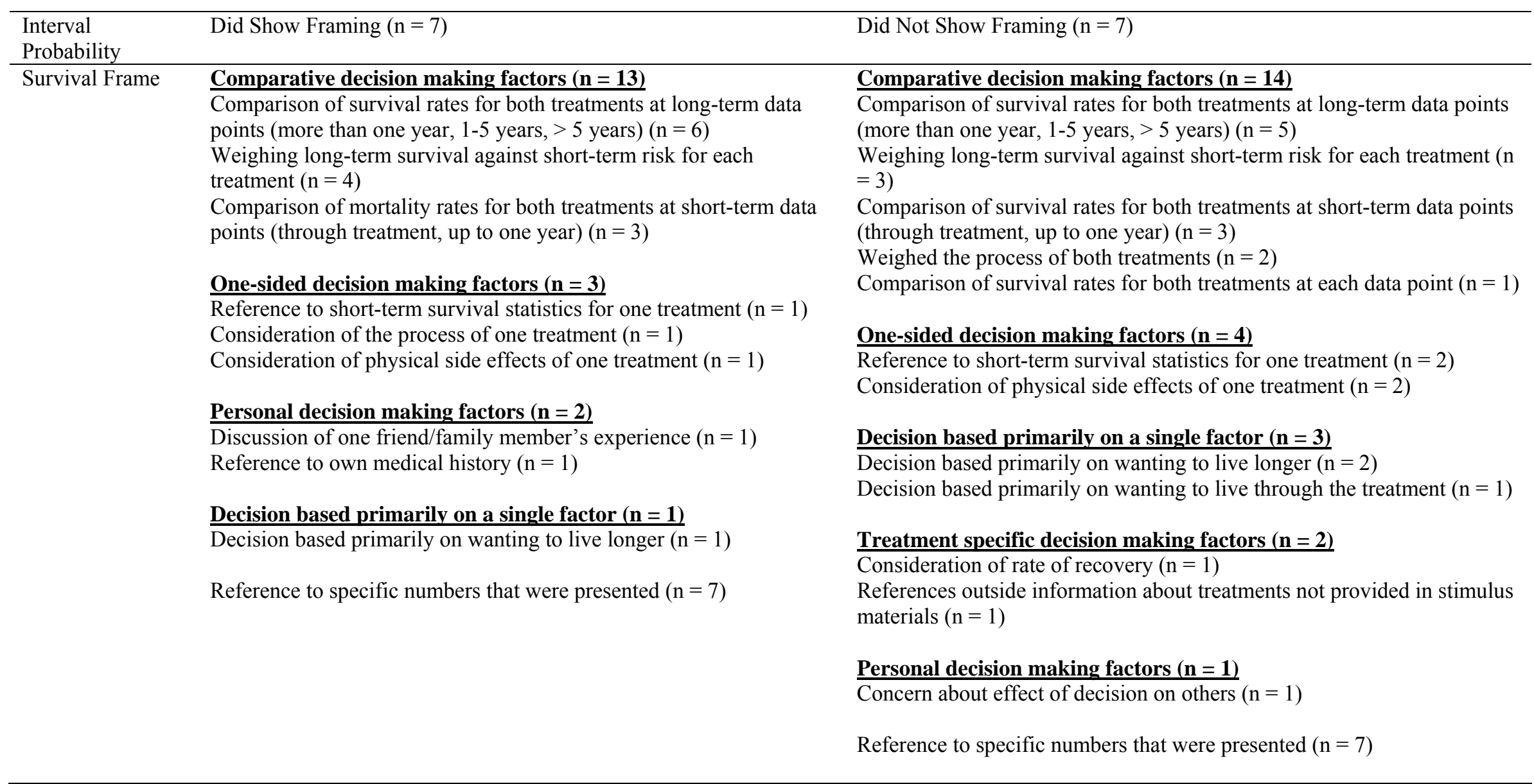




\section{Appendix L (con't)}

Interval

Probability

Mortality Frame
Did Show Framing $(\mathrm{n}=7)$

\section{Decision based primarily on a single factor $(\mathbf{n}=\mathbf{8})$}

Decision based primarily on wanting to live through the treatment (n

$=5$ )

Decision based primarily on wanting to live longer $(\mathrm{n}=2)$

Decision based primarily on certain conditions or qualifications $(\mathrm{n}=$ 1)

\section{Comparative decision making factors $(n=7)$}

Comparison of mortality rates for both treatments at long-term data points (more than one year, $1-5$ years, $>5$ years) $(n=3)$

Comparison of mortality rates for both treatments at short-term data points (through treatment, up to one year) $(n=3)$

Weighing long-term survival against short-term risk for each treatment $(\mathrm{n}=1)$

\section{One-sided decision making factors $(n=1)$}

Reference to short-term mortality statistics for one treatment $(n=1)$

\section{Treatment specific decision making factors $(n=1)$}

References outside information about treatments not provided in

stimulus materials $(\mathrm{n}=1)$

\section{Personal decision making factors $(n=1)$}

Discussion of one friend/family member's experience $(n=1)$

Reference to specific numbers that were presented $(n=5)$
Did Not Show Framing $(\mathrm{n}=7)$

\section{Comparative decision making factors $(\mathrm{n}=9)$}

Comparison of mortality rates for both treatments at short-term data points (through treatment, up to one year) $(n=3)$

Comparison of mortality rates for both treatments at long-term data points (more than one year, $1-5$ years, $>5$ years) $(n=2)$

Comparison of mortality rates for both treatments at each data point (n =2)

Weighed the process of both treatments $(n=1)$

Weighing long-term survival against short-term risk for each treatment $(\mathrm{n}=1)$

\section{Decision based primarily on a single factor $(n=6)$}

Decision based primarily on wanting to live longer $(n=2)$

Decision based primarily on wanting to live through the treatment $(\mathrm{n}=$ 2)

Decision based primarily on certain conditions or qualifications $(n=1)$

Vicarious experience directly related to decisional outcome $(n=1)$

\section{One-sided decision making factors $(n=3)$}

Reference to short-term mortality statistics for one treatment $(n=2)$

Consideration of physical side effects of one treatment $(n=1)$

\section{Treatment specific decision making factors $(n=1)$}

Consideration of rate of recovery $(n=1)$

Reference to specific numbers that were presented $(n=6)$ 


\section{Appendix L (con’t)}

Cumulative

Probability

Survival Frame
Did Show Framing $(\mathrm{n}=16)$

\section{Comparative decision making factors $(\mathrm{n}=33)$}

Comparison of survival rates for both treatments at long-term data points (more than one year, $1-5$ years, $>5$ years) $(n=13)$

Weighing long-term survival against short-term risk for each treatment $(\mathrm{n}=7)$

Comparison of survival rates for both treatments at short-term data points (through treatment, up to one year) $(n=6)$

Weighed physical side effects of both treatments $(n=3)$

Weighed the process of both treatments $(n=2)$

Comparison of mortality rates for both treatments at short-term data points (through treatment, up to one year) $(n=1)$

Comparison of survival rates for both treatments at each data point ( $\mathrm{n}$ $=1)$

\section{One-sided decision making factors $(n=10)$}

Consideration of the process of one treatment $(n=3)$

Reference to long-term survival statistics for one treatment $(n=3)$

Reference to short-term survival statistics for one treatment $(\mathrm{n}=2)$

Consideration of physical side effects of one treatment $(n=2)$

\section{Decision based primarily on a single factor $(n=6)$}

Decision based primarily on wanting to live longer $(n=5)$

Decision based primarily on certain conditions or qualifications $(n=1)$

\section{Treatment specific decision making factors $(n=4)$}

Consideration of rate of recovery $(n=3)$

Consideration of the quality of the hospital and the characteristics of the $\operatorname{staff}(\mathrm{n}=1)$

\section{Personal decision making factors $(n=2)$}

Reference to own medical history $(\mathrm{n}=1)$

Discussion of one friend/family member's experience $(n=1)$

Reference to specific numbers that were presented $(n=13)$
Did Not Show Framing $(\mathrm{n}=14)$

\section{Comparative decision making factors $(\mathrm{n}=\mathbf{1 8})$}

Comparison of survival rates for both treatments at long-term data points (more than one year, $1-5$ years, $>5$ years) $(n=7)$

Weighing long-term survival against short-term risk for each treatment $(\mathrm{n}=4)$

Comparison of survival rates for both treatments at each data point $(\mathrm{n}=$ 3)

Weighed the process of both treatments $(n=2)$

Comparison of survival rates for both treatments at short-term data

points (through treatment, up to one year) $(n=2)$

Decision based primarily on a single factor $(n=9)$

Decision based primarily on wanting to live through the treatment $(\mathrm{n}=$ 4)

Decision based primarily on wanting to live longer $(n=3)$

Decision based primarily on certain conditions or qualifications $(n=1)$

Vicarious experience directly related to decisional outcome $(n=1)$

\section{One-sided decision making factors $(n=7)$}

Consideration of physical side effects of one treatment $(n=3)$

Reference to short-term mortality statistics for one treatment $(\mathrm{n}=2)$

Reference to long-term survival statistics for one treatment $(\mathrm{n}=1)$

Reference to short-term survival statistics for one treatment $(n=1)$

\section{Personal decision making factors $(n=3)$}

Discussion of one friend/family member's experience $(n=3)$

\section{Treatment specific decision making factors $(n=2)$}

Consideration of rate of recovery $(n=2)$

Reference to specific numbers that were presented $(n=10)$ 
Appendix L (con't)

\begin{tabular}{|c|c|c|}
\hline $\begin{array}{l}\text { Cumulative } \\
\text { Probability }\end{array}$ & Did Show Framing $(n=16)$ & Did Not Show Framing $(n=14)$ \\
\hline \multirow[t]{26}{*}{ Mortality Frame } & Comparative decision making factors $(n=18)$ & Comparative decision making factors $(n=13)$ \\
\hline & $\begin{array}{l}\text { Comparison of mortality rates for both treatments at each data point } \\
(\mathrm{n}=7)\end{array}$ & $\begin{array}{l}\text { Comparison of mortality rates for both treatments at each data point (n } \\
=5 \text { ) }\end{array}$ \\
\hline & $\begin{array}{l}\text { Comparison of mortality rates for both treatments at short-term data } \\
\text { points (through treatment, up to one year) }(n=5)\end{array}$ & $\begin{array}{l}\text { Comparison of mortality rates for both treatments at short-term data } \\
\text { points (through treatment, up to one year) }(n=3)\end{array}$ \\
\hline & $\begin{array}{l}\text { Comparison of mortality rates for both treatments at long-term data } \\
\text { points (more than one year, } 1-5 \text { years, }>5 \text { years })(n=4)\end{array}$ & $\begin{array}{l}\text { Weighing long-term survival against short-term risk for each treatment } \\
(\mathrm{n}=2)\end{array}$ \\
\hline & Weighed the process of both treatments $(n=2)$ & $\begin{array}{l}\text { Comparison of mortality rates for both treatments at long-term data } \\
\text { points (more than one year, } 1-5 \text { years, }>5 \text { years })(n=1)\end{array}$ \\
\hline & One-sided decision making factors $(n=7)$ & Weighed physical side effects of both treatments $(n=1)$ \\
\hline & Reference to short-term mortality statistics for one treatment $(\mathrm{n}=2)$ & Weighed the process of both treatments $(n=1)$ \\
\hline & Reference to long-term mortality statistics for one treatment $(n=2)$ & \\
\hline & Consideration of the process of one treatment $(n=2)$ & Decision based primarily on a single factor $(n=12)$ \\
\hline & Consideration of physical side effects of one treatment $(n=1)$ & $\begin{array}{l}\text { Decision based primarily on wanting to live through the treatment }(\mathrm{n}= \\
5)\end{array}$ \\
\hline & Treatment specific decision making factors $(n=6)$ & Decision based primarily on wanting to live longer $(\mathrm{n}=5)$ \\
\hline & Consideration of rate of recovery $(n=3)$ & Decision based primarily on certain conditions or qualifications $(n=2)$ \\
\hline & $\begin{array}{l}\text { Consideration of the quality of the hospital and the characteristics of } \\
\text { the } \operatorname{staff}(n=2)\end{array}$ & One-sided decision making factors $(n=9)$ \\
\hline & Weighing benefits of treatment vs. no treatment vs. alternative & Consideration of the process of one treatment $(n=3)$ \\
\hline & methods $(\mathrm{n}=1)$ & Reference to short-term mortality statistics for one treatment $(n=3)$ \\
\hline & & Reference to long-term mortality statistics for one treatment $(n=1)$ \\
\hline & Decision based primarily on a single factor $(n=4)$ & Consideration of physical side effects of one treatment $(n=1)$ \\
\hline & $\begin{array}{l}\text { Decision based primarily on wanting to live through the treatment ( } \mathrm{n} \\
=3 \text { ) }\end{array}$ & Reference to long-term survival statistics for one treatment $(n=1)$ \\
\hline & Decision based primarily on certain conditions or qualifications $(\mathrm{n}=$ & Treatment specific decision making factors $(n=4)$ \\
\hline & 1) & $\begin{array}{l}\text { References outside information about treatments not provided in } \\
\text { stimulus materials }(\mathrm{n}=3)\end{array}$ \\
\hline & Personal decision making factors $(n=2)$ & Consideration of physician recommendation $(\mathrm{n}=1)$ \\
\hline & $\overline{\text { Discussion of one friend/family member's experience }(n=1)}$ & \\
\hline & Reference to own medical history $(n=1)$ & Personal decision making factors $(n=2)$ \\
\hline & & 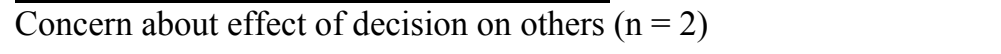 \\
\hline & Reference to specific numbers that were presented $(n=16)$ & \\
\hline & & Reference to specific numbers that were presented $(n=13)$ \\
\hline
\end{tabular}




\section{Appendix M}

Qualitative Results Table for Research Question 4a - Age Differences in Framing

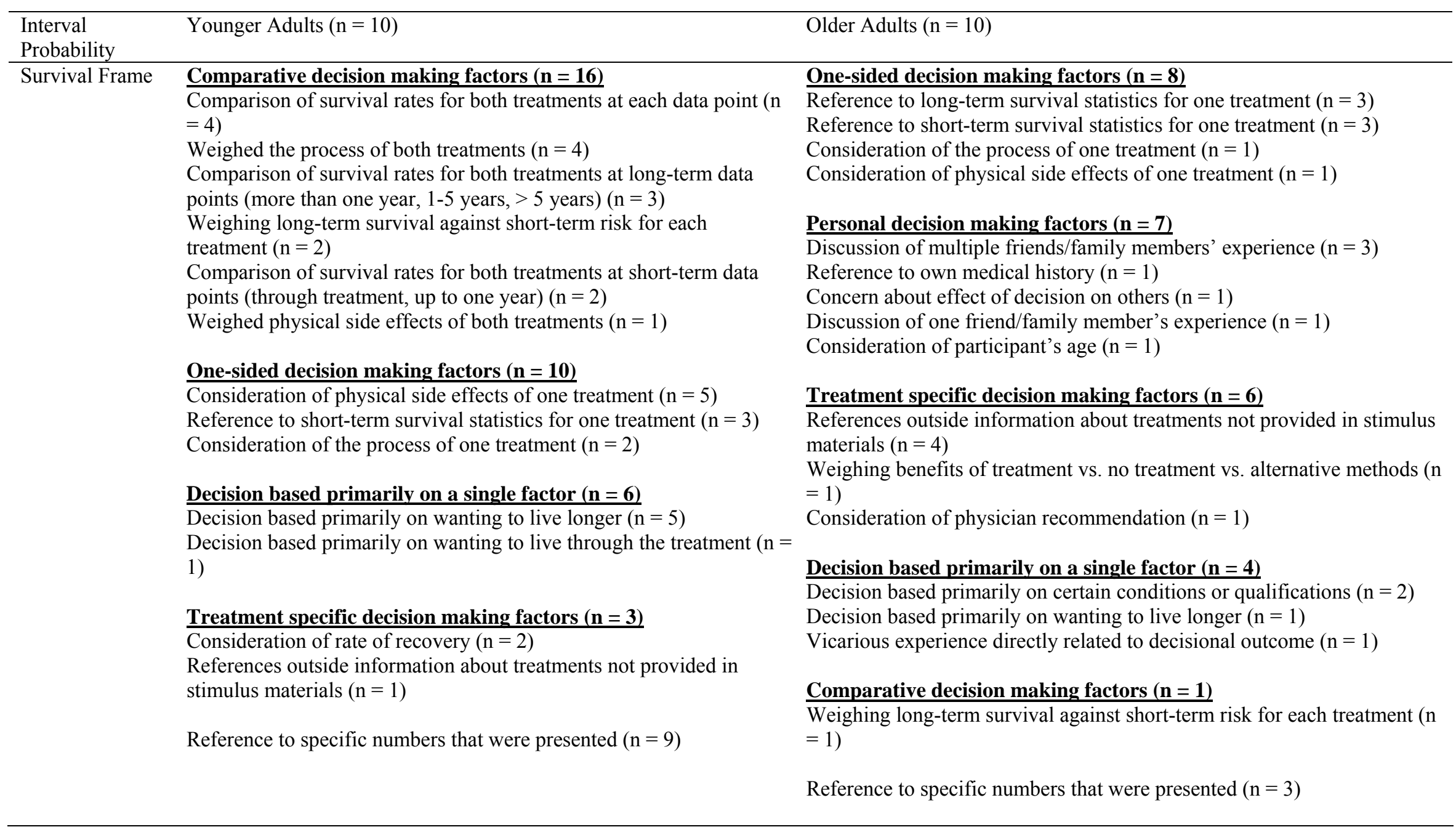




\section{Appendix M (con’t)}

Interval

Probability

Mortality Frame

Older Adults $(\mathrm{n}=10)$

\section{Decision based primarily on a single factor $(n=8)$}

Decision based primarily on wanting to live through the treatment $(\mathrm{n}=3)$

Decision based primarily on certain conditions or qualifications $(n=2)$

Decision based primarily on wanting to live longer $(\mathrm{n}=2)$

Vicarious experience directly related to decisional outcome $(n=1)$

2)

Comparison of mortality rates for both treatments at short-term data

points (through treatment, up to one year) $(n=2)$

Weighed the process of both treatments $(n=1)$

Weighing long-term survival against short-term risk for each treatment $(\mathrm{n}=1)$

\section{Decision based primarily on a single factor $(n=6)$}

Decision based primarily on wanting to live longer $(n=4)$

Decision based primarily on wanting to live through the treatment $(\mathrm{n}=$ 2)

\section{One-sided decision making factors $(n=5)$}

Reference to short-term mortality statistics for one treatment $(n=3)$

Reference to long-term mortality statistics for one treatment $(n=1)$

Consideration of physical side effects of one treatment $(n=1)$

Personal decision making factors $(n=3)$

Discussion of multiple friends/family members' experience $(\mathrm{n}=1)$

Concern about effect of decision on others $(n=1)$

Reference to own medical history $(\mathrm{n}=1)$

\section{Treatment specific decision making factors $(n=1)$}

Consideration of rate of recovery $(n=1)$

Reference to specific numbers that were presented $(n=9)$

\section{Personal decision making factors $(n=7)$}

Discussion of multiple friends/family members' experience $(n=2)$

Reference to own medical history $(\mathrm{n}=2)$

Consideration of participant's age $(n=2)$

Discussion of one friend/family member's experience $(n=1)$

\section{Treatment specific decision making factors $(n=6)$}

Consideration of the quality of the hospital and the characteristics of the staff $(\mathrm{n}=2)$

Consideration of rate of recovery $(n=2)$

References outside information about treatments not provided in stimulus materials $(\mathrm{n}=1)$

Consideration of physician recommendation $(n=1)$

\section{Comparative decision making factors $(n=6)$}

Comparison of mortality rates for both treatments at short-term data points

(through treatment, up to one year) $(\mathrm{n}=2)$

Weighed the process of both treatments $(\mathrm{n}=1)$

Comparison of mortality rates for both treatments at long-term data points (more than one year, 1-5 years, $>5$ years) $(n=1)$

Weighing long-term survival against short-term risk for each treatment $(\mathrm{n}=$ 1)

Weighed physical side effects of both treatments $(n=1)$

\section{One-sided decision making factors $(n=6)$}

Consideration of physical side effects of one treatment $(n=3)$

Reference to short-term mortality statistics for one treatment $(n=2)$

Reference to long-term mortality statistics for one treatment $(n=1)$ 


\section{Appendix N}

Qualitative Results Table for Research Question 4b - Age Differences in Framing

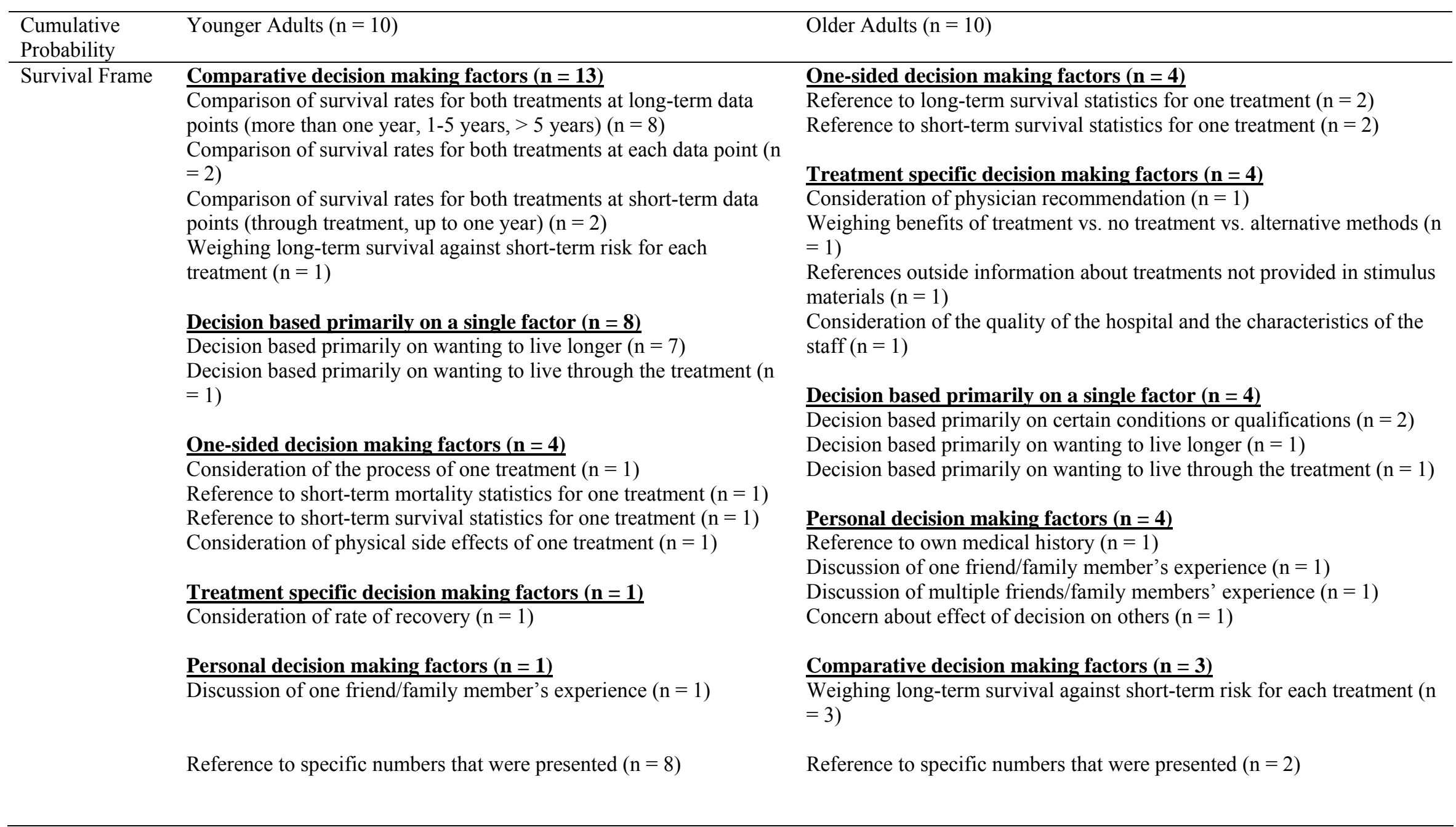




\section{Appendix N (con't)}

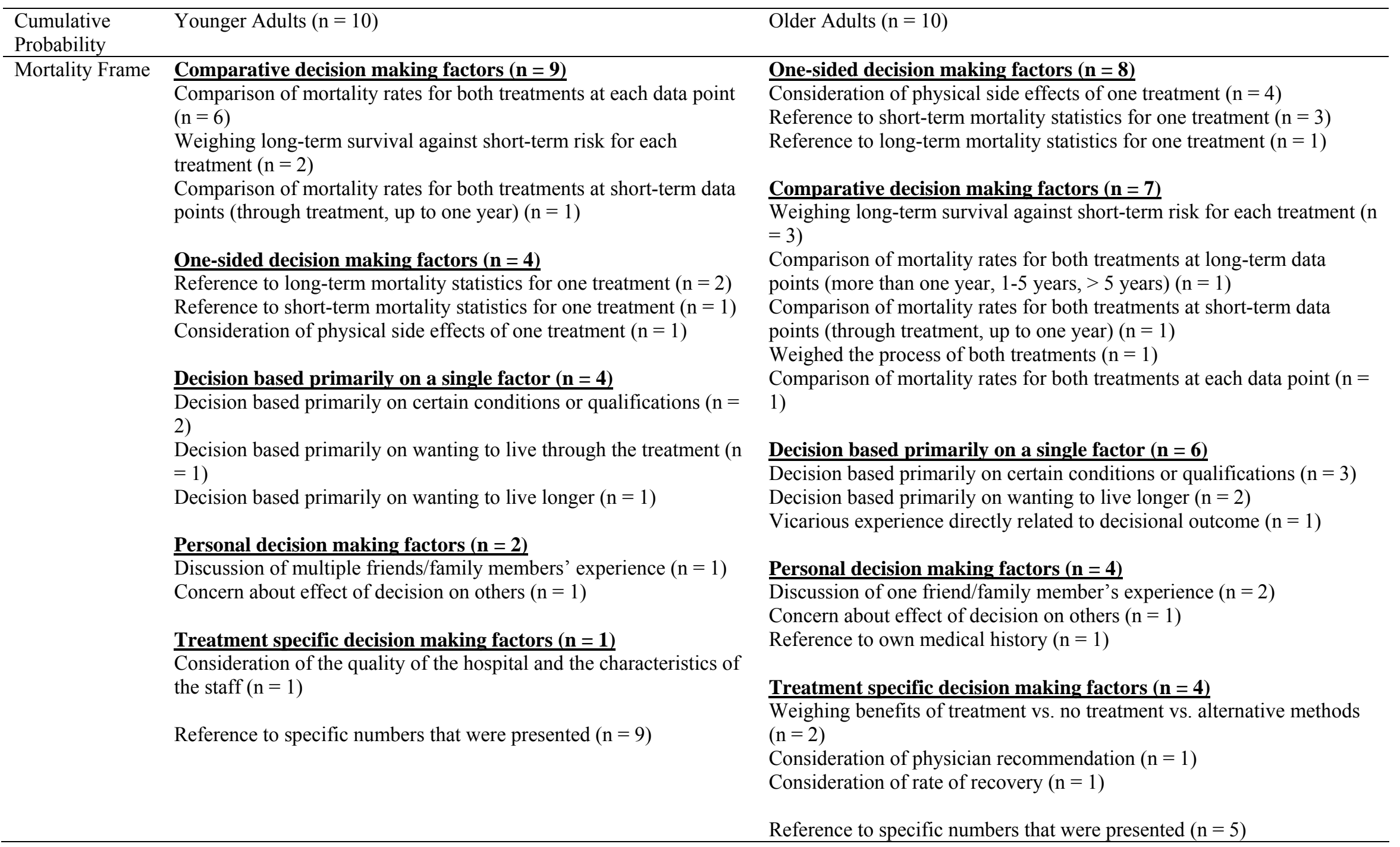




\section{Appendix O}

Results Table from Exploratory Research Question

\begin{tabular}{ll}
\hline Older Adults Who Demonstrated Framing (in & Older Adults Who Did Not Demonstrate \\
either scenario $[\mathrm{n}=15])$ & Framing (in both scenarios $[\mathrm{n}=25])$
\end{tabular}

Living longest at 5-year mark $(n=9)$

Relative/friend/personal experience with cancer treatments $(n=4)$

Quality of life $(\mathrm{n}=2)$

Avoidance of pain and suffering $(\mathrm{n}=2)$

Consideration of my age $(n=2)$

How many people died through the treatment $(n=2)$

Effectiveness/efficiency of results for surgery vs. radiation $(\mathrm{n}=2)$

Recovery time $(\mathrm{n}=1)$

Side effects for surgery vs. radiation $(n=1)$
Relative/friend/personal experience with cancer treatment $(n=12)$

Effectiveness/efficiency of results for surgery vs. radiation $(\mathrm{n}=8)$

Living longest at 5-year mark $(\mathrm{n}=5)$

How many people died through the treatment $(\mathrm{n}=4)$

Recovery time $(\mathrm{n}=3)$

Quality of life $(\mathrm{n}=3)$

Wanting the treatment that is easiest on the body $(\mathrm{n}=2)$

Ability to take care of spouse while receiving treatment $(\mathrm{n}=2)$

Avoidance of pain and suffering $(n=2)$

$\operatorname{Cost}(\mathrm{n}=2)$

Time spent at home during treatment $(n=2)$

Side effects for surgery vs. radiation $(\mathrm{n}=2)$

Consideration of my age $(n=1)$

The severity of the cancer $(n=1)$ 
Appendix O (con't)

Younger Adults Who Demonstrated Framing (in either scenario $[\mathrm{n}=22]$ )
Younger Adults Who Did Not Demonstrate Framing (in both scenarios $[\mathrm{n}=18]$ )
Living longest at 5-year mark $(\mathrm{n}=16)$

How many people died through the treatment $(\mathrm{n}=7)$

Recovery time $(\mathrm{n}=5)$

Relative/friend/personal experience with cancer treatment $(n=4)$

Effectiveness/efficiency of results for surgery vs. radiation $(\mathrm{n}=3)$

Overall mortality rate $(\mathrm{n}=3)$

Side effects for surgery vs. radiation $(n=2)$

Riskiness of surgery $(n=2)$

Overall survival rate $(\mathrm{n}=2)$

Quality of life $(\mathrm{n}=2)$

Wanting the treatment that is easiest on the body $(n=1)$

Consideration of my current health $(n=1)$

Consideration of my age $(\mathrm{n}=1)$

Time spent at home during treatment $(n=1)$

Characteristics of the cancer $(n=1)$
Overall survival rate $(\mathrm{n}=8)$

How many people died through the treatment $(\mathrm{n}=7)$

Living longest at 5-year mark $(\mathrm{n}=6)$

Quality of life $(n=5)$

Side effects for surgery vs. radiation $(n=5)$

Overall mortality rate $(n=3)$

Consideration of the effect of the decision on others $(\mathrm{n}=2)$

Relative/friend/personal experience with cancer treatment $(\mathrm{n}=2)$

Effectiveness/efficiency of results for surgery vs. radiation $(\mathrm{n}=1)$

Time spent at home during treatment $(\mathrm{n}=1)$

Risk of surgery $(\mathrm{n}=1)$

Opinion of physician $(n=1)$ 


\section{Appendix O (con’t)}

Older Adults $(\mathrm{n}=40) \quad$ Younger Adults $(\mathrm{n}=40)$

Relative/friend/personal experience with cancer treatments $(n=16)$

Living longest at 5-year mark $(\mathrm{n}=14)$

Effectiveness/efficiency of results for surgery vs. radiation $(\mathrm{n}=10)$

How many people died through the treatment $(n=6)$

Quality of life $(\mathrm{n}=5)$

Recovery time $(\mathrm{n}=4)$

Avoidance of pain and suffering $(n=4)$

Consideration of my age $(n=3)$

Side effects for surgery vs. radiation $(n=3)$

Wanting the treatment that is easiest on the body $(\mathrm{n}=2)$

Time spent at home during treatment $(n=2)$

Ability to take care of spouse while receiving treatment $(\mathrm{n}=2)$

$\operatorname{Cost}(\mathrm{n}=2)$

The severity of the cancer $(n=1)$
Living longest at 5-year mark $(\mathrm{n}=22)$

How many people died through the treatment $(\mathrm{n}=14)$

Overall survival rate $(\mathrm{n}=10)$

Side effects for surgery vs. radiation $(n=7)$

Quality of life $(n=7)$

Relative/friend/personal experience with cancer treatments $(n=6)$

Overall mortality rate $(n=6)$

Recovery time $(\mathrm{n}=5)$

Risk of surgery $(n=3)$

Effectiveness/efficiency of results for surgery vs. radiation $(n=4)$

Time spent at home during treatment $(\mathrm{n}=$ 2)

Consideration of the effect of the decision on others $(n=2)$

Opinion of physician $(\mathrm{n}=1)$

Consideration of my current health $(\mathrm{n}=1)$

Consideration of my age $(n=1)$

Wanting the treatment that is easiest on the body $(\mathrm{n}=1)$

Characteristics of the cancer $(n=1)$ 